\title{
NASH EQUILIBRIUM FOR A MULTIOBJECTIVE CONTROL PROBLEM RELATED TO WASTEWATER MANAGEMENT ${ }^{*} * *$
}

\author{
NÉSTOR García-Chan ${ }^{1}$, RAFAEl MuÑOZ-SOlA ${ }^{1}$ \\ And Miguel ERnesto VÁzQUez-MÉndez ${ }^{2}$
}

\begin{abstract}
This paper is concerned with mathematical modelling in the management of a wastewater treatment system. The problem is formulated as looking for a Nash equilibrium of a multiobjective pointwise control problem of a parabolic equation. Existence of solution is proved and a first order optimality system is obtained. Moreover, a numerical method to solve this system is detailed and numerical results are shown in a realistic situation posed in the estuary of Vigo (Spain).
\end{abstract}

Mathematics Subject Classification. 49J20, 49K20, 90C29, 91B76

Received January 31, 2007. Revised July 18, 2007 and October 12, 2007.

Published online March 6, 2008.

\section{INTRODUCTION}

Management, treatment and elimination of waste are one of the most important environmental problems. In the particular case of wastewater, a widespread solution consists of discharging it into rivers or directly into the sea. Current legislation in developed countries requires that wastewater be treated in a purifying plant before being discharged. These treatments can be not only necessary but also very expensive, and its management involves environmental and economical aspects. The problem becomes more complicated when several purifying plants are going to discharge wastewater into the same domain (a lake, an estuary, ...).

In the last years, several works (see, for example, $[2-4,12,13]$ ) have used mathematical models and optimal control techniques to solve this type of problems. In [12], a wastewater treatment system consisting of several purifying plants discharging into the same domain is considered and, by assuming that every plant is controlled by a unique organization, the management of the treatment system is formulated and studied as an optimal control problem with state and control pointwise constraints. The main goal of this work consists of studying the problem when each purifying plant is controlled by a different organization (industries, municipal

Keywords and phrases. Optimal control, pointwise control, Nash equilibrium, existence, optimality conditions, numerical simulation, wastewater management.

* The research contained in this work was supported by Project MTM2006-01177 of Ministerio de Educación y Ciencia (Spain).

** First author is thankful for financial support from Programa AlBan of European Union (code E04D045803MX) and CONACyT of Mexico (code 165721).

1 Departamento de Matemática Aplicada, Universidad de Santiago de Compostela, Facultad de Matemáticas, 15782 Santiago de Compostela, Spain. netog-g@hotmail.com; rafams@usc.es

2 Departamento de Matemática Aplicada, Universidad de Santiago de Compostela, Escola Politécnica Superior, 27002 Lugo, Spain. ernesto@usc.es 


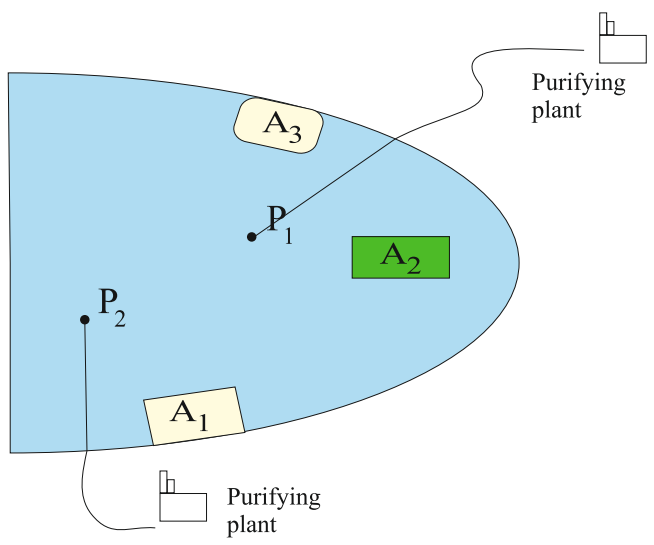

Figure 1. Scheme of domain $\Omega$.

governments, ...). In this case, the problem has to be formulated in the framework of the multiobjective control problems and noncooperative games theory.

In a multiobjective control problem the minimization of some functionals depending on some controls is considered. At first glance, the goal could be to obtain the controls minimizating simultaneously every functional. However, in the general case, there are not those controls and the objective has to be formulated in other terms. There are several strategies in order to choose the controls, depending on the character of the problem. These strategies can be cooperative (when the controls cooperate between them in order to achieve the goals) and noncooperative. We are going to use the Nash equilibrium, which define a multiobjective optimization strategy in noncooperative problems. Essentially, a number of players equal to the number of controls is considered (in our case, the players are the different organizations controlling every purifying plant). For each player, we consider a functional to minimize and a strategy space defined as the set which the corresponding control belongs to. A strategy for every player is looked for, in such a way that no player could change its strategy without increase its functional, if the other players do not change their strategies. In a formal way, we can give the following definition [8]:

Definition 1.1. Let be a multiobjective control problem with controls $u_{1}, \ldots, u_{G}$ and functionals to minimize $J_{i}=J_{i}\left(u_{1}, \ldots, u_{G}\right), i=1, \ldots, G$. Let be $M_{i}$ the admissible control set (strategy space) for the $i$-th control. The element $\left(u_{1}^{*}, \ldots, u_{G}^{*}\right) \in \prod_{i=1}^{G} M_{i}$ is a Nash equilibrium of the problem if the following equality holds

$$
J_{i}\left(u_{1}^{*}, \ldots, u_{i-1}^{*}, u_{i}^{*}, u_{i+1}^{*}, \ldots, u_{G}^{*}\right)=\min _{u_{i} \in M_{i}} J_{i}\left(u_{1}^{*}, \ldots, u_{i-1}^{*}, u_{i}, u_{i+1}^{*}, \ldots, u_{G}^{*}\right)
$$

for all $i=1, \ldots, G$.

In the next section we are going to formulate the problem of the management of a wastewater treatment system in terms of finding a Nash equilibrium for a multiobjective control problem of parabolic type. In Section 3 we do a mathematical analysis of the problem. In Section 4 we prove the existence of a Nash equilibrium. In Section 5 we give a first order optimality system. In Section 6 we propose a numerical algorithm to solve the problem. Finally, in Section 7, we present the numerical results obtained in a realistic situation posed in the estuary of Vigo (Spain).

\section{Mathematical Formulation}

We consider a bounded domain $\Omega \subset \mathbb{R}^{2}$ occupied by shallow waters, where polluting wastewaters are discharged through $N_{E}$ submarine outfalls located at points $P_{j} \in \Omega, j=1, \ldots, N_{E}$ and connected to their respective purifying plants located on the coast (see Fig. 1). Moreover, we assume the existence of several areas $A_{l} \subset \Omega$, 
$l=1, \ldots, N_{Z}$, representing fisheries, beaches or marine recreation zones, where it is necessary to guarantee a water quality with pollution levels lower than some allowed threshold levels (an indicator of the water quality is fixed and its concentration in the $l$-th zone must be lower than a maximum value $\sigma_{l}$ ). In order to do it, we assume that the $j$-th plant is taking care of $n_{j}$ zones and a penalty is imposed on that plant if the concentration of indicator in one of its zones is greater than the threshold level. Usually each area is controlled by only one plant and then $\sum_{j=1}^{n_{N_{E}}} n_{j}=N_{Z}$, but the case $\sum_{j=1}^{n_{N_{E}}} n_{j}>N_{Z}$, several plants controlling common areas, is also permitted. Obviously, the manager of each purifying plant looks for a purification strategy which minimize the sum of penalties and cost purification and we look for a whole strategy accepted by all plant managers.

State system. The first step to formulate this problem is to choose an indicator of water quality and to provide a mathematical model which gives, from the discharges at $P_{j} \in \Omega, j=1, \ldots, N_{E}$, the indicator concentration in $\Omega$. By assuming domestic (no industrial) discharges, we take faecal coliphorm (CF) as indicator of water quality. $\mathrm{CF}$ concentration in a shallow water domain is given by (see, for instance [5])

$$
\left.\begin{array}{ll}
\frac{\partial \rho}{\partial t}+\vec{u} \cdot \nabla \rho-\beta \Delta \rho+\kappa \rho=\frac{1}{h}\left[\sum_{j=1}^{N_{E}} m_{j}(t) \delta\left(x-P_{j}\right)\right] & \text { in } \Omega \times(0, T) \\
\rho(x, 0)=\rho_{0}(x) & \text { in } \Omega \\
\frac{\partial \rho}{\partial n}=0 & \text { on } \partial \Omega \times(0, T)
\end{array}\right\}
$$

where $\rho$ is depth-averaged CF concentration, $h \in C(\bar{\Omega} \times[0, T])$ is the height of water (which we suppose that satisfies $h(x, t) \geq \alpha>0, \forall(x, t) \in \bar{\Omega} \times[0, T]), \vec{u} \in\left[L^{\infty}\left(0, T ; W^{1, \infty}(\Omega)\right)\right]^{2}$ is the depth-averaged horizontal velocity of water, $\beta>0$ is a viscosity coefficient collecting turbulent and dispersion effects, $\kappa \in \mathbb{R}$ is an experimental coefficient related to the loss rate of $\mathrm{CF}, m_{j} \in L^{\infty}(0, T)$ is the mass flow rate of $\mathrm{CF}$ discharged at $P_{j}, \delta\left(x-P_{j}\right)$ denotes the Dirac measure at $P_{j}$ and $\rho_{0} \in C(\bar{\Omega})$ is the initial CF concentration.

Controls. In this model (system (2.1)), each purifying plant has one control associated with it. In effect, the management of the purification in a plant is equivalent to determine the amount of $\mathrm{CF}$ discharged after purification and then, the control associated with the $j$-th plant is the function $m_{j}(t)$.

Objective functionals. The functional to minimize corresponding to the $j$-th plant, as we have said, must collect two different aspects: purification process cost and cost because of insufficient purification. Firstly, we assume that the purification process cost in a particular plant is known and dependent on CF discharge through the corresponding outfall, in a similar way as it is shown in Figure 2. Moreover, if we denote by $\bar{m}_{j}$ the maximum mass flow rate of CF arriving to the $j$-th plant and $\underline{m}_{j}$ the minimum mass flow rate of CF discharging at $P_{j}$ (corresponding with the maximum purification in that plant) we also suppose that $f_{j} \in C^{2}\left[\underline{m}_{j}, \bar{m}_{j}\right]$ is strictly convex in this interval. Secondly, if the purification in the $j$-th plant is not sufficient and the CF concentration in its zones is greater than the corresponding threshold $\left(\sigma_{i}\right.$ in the zone $\left.A_{i}\right)$, a penalty is imposed on that plant. We assume that penalty amount is an increasing function of CF extra concentration.

According to this, if we define $n_{0}=0$ and we number the protected areas in such a way that for $j=$ $1,2, \ldots, N_{E}$, the $j$-th plant takes care of $\cup_{i=n_{(j-1)}+1}^{n_{j}} A_{i}$, the objective function to minimize corresponding to the $j$-th plant is given by

$$
J_{j}\left(m_{1}, m_{2}, \ldots, m_{N_{E}}\right)=\int_{0}^{T} f_{j}\left(m_{j}(t)\right) \mathrm{d} t+\sum_{i=n_{(j-1)}+1}^{n_{j}} \frac{1}{\epsilon_{i}} \int_{\bar{A}_{i} \times(0, T)} \psi\left(\rho(x, t)-\sigma_{i}\right) \mathrm{d} x \mathrm{~d} t
$$




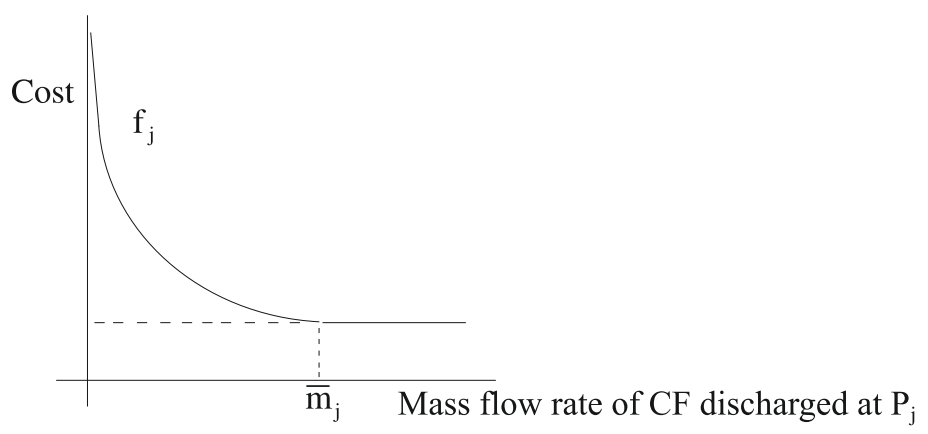

FiguRE 2. Standard shape for function giving the purification cost of the $j$-th plant.

where $f_{j}$ is a known function giving the purification cost of the $j$-th plant, $\epsilon_{l}>0, l=1,2, \ldots, N_{Z}$ is a penalty parameter and, for a fixed $\delta>0, \psi(y)$ is a $C^{2}$ regularization of $\frac{1}{2} y_{+}^{2}$, where $y_{+}$stands for the "positive part" function, that is

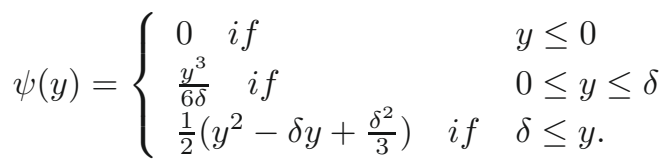

Remark 2.1. The case of an area controlled by several plants can be handled by labelling this area as many times as the number of plants controlling it. In this way, the number of areas would became $\sum_{j=1}^{n_{N_{E}}} n_{j}$ instead of $N_{Z}$.

Strategy spaces and multiobjective control problem. For $j=1,2, \ldots, N_{E}$ we denote by $M_{j}=\{m \in$ $L^{\infty}(0, T) ; 0<\underline{m}_{j} \leq m(t) \leq \bar{m}_{j}$, a.e. in $\left.(0, T)\right\}$ the strategy space for the $j$-th plant and we consider the following multiobjective control problem:

Problem $(\mathcal{P})$ : For $j=1, \ldots, N_{E}$, find the function $m_{j}(t) \in M_{j}$ which minimize the functional $J_{j}\left(m_{1}, \ldots, m_{N_{E}}\right)$ given by $(2.2)$, where $\rho(x, t)$ is the solution of system $(2.1)$.

Goal. We look for a purification strategy for every plant accepted by all plant managers in the sense that none could change its strategy without increase its objective functional, if the others do not change their strategies. According to Definition 1.1 this is equivalent to find a Nash equilibrium for the problem $(\mathcal{P})$, that is:

Find a set of controls $\left(m_{1}^{*}, \ldots, m_{N_{E}}^{*}\right) \in \prod_{j=1}^{N_{E}} M_{j}$ such that, for $j=1,2, \ldots, N_{E}$

$$
J_{j}\left(m_{1}^{*}, \ldots, m_{j-1}^{*}, m_{j}^{*}, m_{j+1}^{*}, \ldots, m_{N_{E}}^{*}\right)=\min _{m_{j} \in M_{j}} J_{j}\left(m_{1}^{*}, \ldots, m_{j-1}^{*}, m_{j}, m_{j+1}^{*}, \ldots, m_{N_{E}}^{*}\right)
$$

\section{Mathematical ANALYsis}

\subsection{The state system}

The solution of system (2.1) can be defined by transposition techniques (see $[7,11,12])$ in the following way:

Definition 3.1. Given $r, s \in[1,2), \frac{2}{r}+\frac{2}{s}>3$, we say that $\rho \in L^{r}\left(0, T ; W^{1, s}(\Omega)\right)$ is a solution of the problem (2.1) if for all $\Phi \in C^{1}(\bar{\Omega} \times[0, T])$ such that $\Phi(., T)=0$ it is satisfied that:

$$
\int_{0}^{T} \int_{\Omega}\left(-\frac{\partial \Phi}{\partial t} \rho+\beta \nabla \Phi \cdot \nabla \rho+\vec{u} \Phi \cdot \nabla \rho+\kappa \Phi \rho\right) \mathrm{d} x \mathrm{~d} t=\sum_{j=1}^{N_{E}} \int_{0}^{T} \frac{1}{h\left(P_{j}, t\right)} \Phi\left(P_{j}, t\right) m_{j}(t) \mathrm{d} t+\int_{\Omega} \Phi(x, 0) \rho_{0}(x) \mathrm{d} x
$$


Let $\mathcal{A}$ be the differential operator defined by

$$
\mathcal{A} w=-\beta \Delta w+\vec{u} \cdot \nabla w+\kappa w .
$$

We denote by $\mathcal{A}^{*}$ its formal adjoint,

$$
\mathcal{A}^{*} \Phi=-\beta \Delta \Phi-\operatorname{div}(\Phi \vec{u})+\kappa \Phi
$$

and by $\frac{\partial \Phi}{\partial n_{\mathcal{A}^{*}}}$ the conormal derivative operator associated to $\mathcal{A}^{*}$, that is,

$$
\frac{\partial \Phi}{\partial n_{\mathcal{A}^{*}}}=\beta \frac{\partial \Phi}{\partial n}+\Phi \vec{u} \cdot \vec{n}
$$

We also assume that $\Omega$ is a bounded domain with smooth enough boundary satisfying the condition (cf. [9], p. 9):

C1. There are two constants $\alpha^{*} \in(0,1)$ and $r_{0}>0$ such that for all $x_{0} \in \partial \Omega$ and for all $r \in\left(0, r_{0}\right]$, $\operatorname{meas}\left(B\left(x_{0}, r\right) \cap \Omega\right) \leq\left(1-\alpha^{*}\right) \pi r^{2}$.

Then we have the following result:

Theorem 3.2. There exists a unique function $\rho \in\left[L^{r}\left(0, T ; W^{1, s}(\Omega)\right) \cap L^{2}\left(0, T ; L^{2}(\Omega)\right)\right]$ with $\frac{\partial \rho}{\partial t} \in$ $L^{r}\left(0, T ;\left(W^{1, s^{\prime}}(\Omega)\right)^{\prime}\right)$ such that $\rho$ is a solution of $(2.1)$ and satisfies

$$
\int_{0}^{T}\left\langle-\frac{\partial \Phi}{\partial t}+\mathcal{A}^{*} \Phi, \rho\right\rangle \mathrm{d} t=\sum_{j=1}^{N_{E}} \int_{0}^{T} \frac{1}{h\left(P_{j}, t\right)} \Phi\left(P_{j}, t\right) m_{j}(t) \mathrm{d} t+\int_{\Omega} \Phi(x, 0) \rho_{0}(x) \mathrm{d} x
$$

for all $\Phi \in \mathcal{B}=\left\{\Phi \in L^{2}\left(0, T ; H^{2}(\Omega)\right) \cap H^{1}\left(0, T ; L^{2}(\Omega)\right) ; \frac{\partial \Phi}{\partial n_{\mathcal{A}^{*}}}=0\right.$ on $\left.\partial \Omega \times(0, T), \Phi(., T)=0\right\}$.

Besides:

(1) There exist positive constants $C_{1}, C_{2}, C_{3}, C_{4}$ only depending on data, such that:

$$
\begin{aligned}
& \|\rho\|_{L^{r}\left(0, T ; W^{1, s}(\Omega)\right)} \leq C_{1} \sum_{j=1}^{N_{E}}\left\|m_{j}\right\|_{L^{\infty}(0, T)}+C_{2}\left\|\rho_{0}\right\|_{C(\bar{\Omega})} \\
& \|\rho\|_{L^{2}\left(0, T ; L^{2}(\Omega)\right)} \leq C_{3} \sum_{j=1}^{N_{E}}\left\|m_{j}\right\|_{L^{\infty}(0, T)}+C_{4}\left\|\rho_{0}\right\|_{C(\bar{\Omega})} .
\end{aligned}
$$

(2) If there exists a closed set $E \subset \Omega$ such that $\Omega \backslash E$ is a domain with boundary smooth enough, $\left\{P_{1}, P_{2}, \ldots, P_{N_{E}}\right\} \subset E$ and $\cup_{i=1}^{N_{Z}} \bar{A}_{i} \subset(\Omega \backslash E)$, then:

(a) Function $\rho_{\mid \cup_{i=1}^{N_{Z}} \bar{A}_{i} \times[0, T]} \in C\left(\cup_{i=1}^{N_{Z}} \bar{A}_{i} \times[0, T]\right)$ and there exist constants $C_{5}, C_{6}>0$, such that

$$
\|\rho\|_{C\left(\cup_{i=1}^{N_{Z}} \bar{A}_{i} \times[0, T]\right)} \leq C_{5} \sum_{i=1}^{N_{E}}\left\|m_{i}\right\|_{L^{\infty}(0, T)}+C_{6}\left\|\rho_{0}\right\|_{C(\bar{\Omega})} .
$$

(b) If the initial condition $\rho_{0}=0$ then $\rho \in C^{\alpha}\left(\cup_{i=1}^{N_{Z}} \bar{A}_{i} \times[0, T]\right)$ for some $\alpha \in(0,1)$, and there exists a constant $C_{7}>0$ such that

$$
\|\rho\|_{C^{\alpha}\left(\cup_{i=1}^{N_{Z}} \bar{A}_{i} \times[0, T]\right)} \leq C_{7} \sum_{i=1}^{N_{E}}\left\|m_{i}\right\|_{L^{\infty}(0, T)} .
$$


(c) The mapping

$$
\begin{array}{ccc}
F:\left(L^{\infty}(0, T)\right)^{N_{E}} & \longrightarrow & C\left(\cup_{i=1}^{N_{Z}} \bar{A}_{i} \times[0, T]\right) \\
m=\left(m_{1}, m_{2}, \ldots, m_{N_{E}}\right) & \longrightarrow & F(m)=\rho_{\mid \cup_{i=1}^{N_{Z}} \bar{A}_{i} \times[0, T]}
\end{array}
$$

is well defined and it is affine and continuous.

The proof of the existence and uniqueness of solution and item (1) can be seen in Theorem 3.2 of [12], item (2)(a) is obtained from Lemmas 3.3 and 3.4 of [12], item (2)(b) arises from Theorem 10.1 of Chapter III of [9] and, finally, item (2)(c) is an easy consequence of item (2)(a).

\subsection{The objective functionals}

From Theorem 3.2, the objective functional formally introduced by (2.2) is well defined on $\prod_{j=1}^{N_{E}} M_{j}$ and can be written as

$$
J_{j}\left(m_{1}, m_{2}, \ldots, m_{N_{E}}\right)=\Theta_{j}\left(m_{j}\right)+H_{j}\left(F\left(m_{1}, m_{2}, \ldots, m_{N_{E}}\right)\right)
$$

where

- $\Theta_{j}: M_{j} \longrightarrow \mathbb{R}$ is given by $\Theta_{j}\left(m_{j}\right)=\int_{0}^{T} f_{j}\left(m_{j}(t)\right) \mathrm{d} t$

- $H_{j}: C\left(\cup_{i=1}^{N_{Z}} \bar{A}_{i} \times[0, T]\right) \longrightarrow \mathbb{R}$ is defined by

$$
H_{j}(\rho)=\sum_{i=n_{(j-1)}+1}^{n_{j}} \frac{1}{\epsilon_{i}} \int_{\bar{A}_{i} \times(0, T)} \psi\left(\rho(x, t)-\sigma_{i}\right) \mathrm{d} x \mathrm{~d} t
$$

Lemma 3.3. If $f_{j} \in C^{1}\left[\underline{m}_{j}, \bar{m}_{j}\right]$ then $\Theta_{j}$ is Gateaux differentiable at every point $m_{j} \in M_{j}$ and

$$
\left\langle\Theta_{j}^{\prime}\left(m_{j}\right), \delta m_{j}\right\rangle=\int_{0}^{T} f_{j}^{\prime}\left(m_{j}(t)\right)\left(\delta m_{j}\right)(t) \mathrm{d} t
$$

for all $\delta m_{j} \in L^{\infty}(0, T)$ satisfying $m_{j}+\epsilon_{0} \delta m_{j} \in M_{j}$ for some $\epsilon_{0}>0$.

Proof. Let $m_{j} \in M_{j}$ and let $\delta m_{j} \in L^{\infty}(0, T)$ such that $m_{j}+\epsilon_{0} \delta m_{j} \in M_{j}$ for some $\epsilon_{0}>0$. For each $\lambda \in\left(0, \epsilon_{0}\right]$, we have $m_{j}+\lambda \delta m_{j} \in M_{j}$ and

$$
\frac{\Theta_{j}\left(m_{j}+\lambda \delta m_{j}\right)-\Theta_{j}\left(m_{j}\right)}{\lambda}=\int_{0}^{T} \frac{f_{j}\left(m_{j}(t)+\lambda\left(\delta m_{j}\right)(t)\right)-f_{j}\left(m_{j}(t)\right)}{\lambda} \mathrm{d} t .
$$

For a.e. $t \in(0, T)$, we have

$$
\lim _{\lambda \rightarrow 0^{+}} \frac{f_{j}\left(m_{j}(t)+\lambda\left(\delta m_{j}\right)(t)\right)-f_{j}\left(m_{j}(t)\right)}{\lambda}=f_{j}^{\prime}\left(m_{j}(t)\right)\left(\delta m_{j}\right)(t),
$$

and for $\lambda \in\left(0, \epsilon_{0}\right]$

$$
\frac{f_{j}\left(m_{j}(t)+\lambda\left(\delta m_{j}\right)(t)\right)-f_{j}\left(m_{j}(t)\right)}{\lambda}=f_{j}^{\prime}\left(m_{j}(t)+\theta(t, \lambda) \lambda\left(\delta m_{j}\right)(t)\right)\left(\delta m_{j}\right)(t),
$$


with $\theta(t, \lambda) \in(0,1)$. Hence

$$
\left|\frac{f_{j}\left(m_{j}(t)+\lambda\left(\delta m_{j}\right)(t)\right)-f_{j}\left(m_{j}(t)\right)}{\lambda}\right| \leq C=\left\|\delta m_{j}\right\|_{L^{\infty}(0, T)} \max _{m_{j} \in\left[\underline{m_{j}}, \bar{m}_{j}\right]}\left|f_{j}^{\prime}\left(m_{j}\right)\right| .
$$

Using Lebesgue's dominated convergence theorem, we deduce that $\Theta_{j}$ is Gateaux differentiable at $m_{j}$ and that equation (3.8) holds.

If the function $f_{j}$ is continuous and strictly convex, the functional $\Theta_{j}$ also it is. Moreover, since $\psi$ is convex and differentiable, $H_{j}$ is convex, continuous and Gateaux differentiable. Finally, since the mapping $F$ is affine and continuous (see Th. 3.2), we have the following result:

Theorem 3.4. If $f_{j} \in C^{1}\left[\underline{m}_{j}, \bar{m}_{j}\right]$ is strictly convex, then the functional

$$
m_{j} \in M_{j} \longrightarrow J_{j}\left(m_{1}, \ldots, m_{j-1}, m_{j}, m_{j+1}, \ldots, m_{N_{E}}\right) \in \mathbb{R}
$$

given by (3.7), is continuous, Gateaux differentiable and strictly convex.

\section{Existence of A NASH EQUILIBRIUm}

Results in this section only require $f_{j} \in C\left[\underline{m}_{j}, \bar{m}_{j}\right]$ and strictly convex for all $j=1, \ldots, N_{E}$.

Given $m=\left(m_{1}, \ldots, m_{N_{E}}\right) \in \prod_{j=1}^{N_{E}} M_{j}$ and $\rho_{0} \in C(\bar{\Omega})$ we denote by $\varrho\left(m, \rho_{0}\right)=\rho$, the solution of the problem (2.1) given by Theorem 3.2 (with this notation $\left.F(m)=\varrho\left(m, \rho_{0}\right)_{\mid \cup_{i=1}^{N} \bar{A}_{i} \times[0, T]}\right)$.

Lemma 4.1. Let $\left\{m^{n}\right\} \subset \prod_{j=1}^{N_{E}} M_{j}$ be such that $\left\{m^{n}\right\} \rightarrow m^{*}$ weakly in $\left(L^{\infty}(0, T)\right)^{N_{E}}$. Then, $\forall r, s \in[1,2)$ such that $\frac{2}{r}+\frac{2}{s}>3$,

$$
\begin{aligned}
\varrho\left(m^{n}, \rho_{0}\right) & \rightarrow \varrho\left(m, \rho_{0}\right) \text { weakly in } L^{r}\left(0, T ; W^{1, s}(\Omega)\right) \\
\varrho\left(m^{n}, \rho_{0}\right) & \rightarrow \varrho\left(m, \rho_{0}\right) \text { weakly in } L^{2}\left(0, T ; L^{2}(\Omega)\right) \\
\varrho\left(m^{n}, \rho_{0}\right)_{\mid \cup_{i=1}^{N_{Z}} \bar{A}_{i} \times[0, T]} & \rightarrow \varrho\left(m, \rho_{0}\right)_{\mid \cup_{i=1}^{N_{Z}} \bar{A}_{i} \times[0, T]} \text { in } C\left(\cup_{i=1}^{N_{Z}} \bar{A}_{i} \times[0, T]\right) .
\end{aligned}
$$

Proof. Since $\varrho\left(m, \rho_{0}\right)=\varrho(m, 0)+\varrho\left(0, \rho_{0}\right)$, it suffices to consider the case $\rho_{0}=0$.

Given $r, s \in[1,2)$ such that $\frac{2}{r}+\frac{2}{s}>3$, as the sequence $\left\{m^{n}\right\}$ is bounded in $\left(L^{\infty}(0, T)\right)^{N_{E}}$, we deduce from Theorem 3.2 that $\varrho\left(m^{n}, 0\right)$ is bounded in $L^{2}\left(0, T ; L^{2}(\Omega)\right)$ and in $L^{r}\left(0, T ; W^{1, s}(\Omega)\right)$. This ensures the existence of a subsequence, still denoted $\left\{m^{n}\right\}$, and a function $\bar{\rho} \in L^{r}\left(0, T ; W^{1, s}(\Omega)\right) \cap L^{2}\left(0, T ; L^{2}(\Omega)\right)$ such that

$$
\begin{gathered}
\varrho\left(m^{n}, 0\right) \rightarrow \bar{\rho} \text { weakly in } L^{r}\left(0, T ; W^{1, s}(\Omega)\right) \\
\varrho\left(m^{n}, 0\right) \rightarrow \bar{\rho} \text { weakly in } L^{2}\left(0, T ; L^{2}(\Omega)\right) .
\end{gathered}
$$

Passing to the limit in equations (3.1) and (3.5), we deduce that $\bar{\rho}=\varrho(m, 0)$. The uniqueness of the limit implies that these convergences hold for the whole sequence (and not just a subsequence). Thus we obtain (4.1) and (4.2).

In the same way, since $\varrho\left(m^{n}, 0\right)_{\mid \cup_{i=1}^{N_{Z}} \bar{A}_{i} \times[0, T]}$ is bounded in $C^{\alpha}\left(\cup_{i=1}^{N_{Z}} \bar{A}_{i} \times[0, T]\right)$ and the injection of this space into $C\left(\cup_{i=1}^{N_{Z}} \bar{A}_{i} \times[0, T]\right)$ is compact, there exists a subsequence, still denoted $\left\{m^{n}\right\}$, such that

$$
\varrho\left(m^{n}, 0\right)_{\mid \cup_{i=1}^{N} \bar{A}_{i} \times[0, T]} \rightarrow \varrho(m, 0)_{\mid \cup_{i=1}^{N_{Z}} \bar{A}_{i} \times[0, T]} \text { in } C\left(\cup_{i=1}^{N_{Z}} \bar{A}_{i} \times[0, T]\right) .
$$

Using again the uniqueness of the limit, we obtain (4.3). 
Remark 4.2. Since $M_{j}$ is a bounded subset of $L^{\infty}(0, T)$, the weak $L^{\infty}(0, T)$ topology and the weak $L^{2}(0, T)$ topology coincide over $M_{j}$.

Since the functional (3.13) is strictly convex and weakly lower semicontinuous and the set $M_{j}$ is nonempty, convex and closed in $L^{2}(0, T)$, a classical result of control theory (see for instance [10]) guarantees that, for any fixed $\left(m_{1}, \ldots, m_{j-1}, m_{j+1}, \ldots, m_{N_{E}}\right) \in \prod_{\substack{i=1 \\ i \neq j}}^{N_{E}} M_{i}$ there exists a unique $m_{j}^{*} \in M_{j}$ such that

$$
J_{j}\left(m_{1}, \ldots, m_{j-1}, m_{j}^{*}, m_{j+1}, \ldots, m_{N_{E}}\right)=\min _{m_{j} \in M_{j}} J_{j}\left(m_{1}, \ldots, m_{j-1}, m_{j}, m_{j+1}, \ldots, m_{N_{E}}\right) .
$$

This enables us to define the mapping

$$
\begin{aligned}
& S_{j}: \quad \prod_{\substack{i=1 \\
i \neq j}}^{N_{E}} M_{i} \quad \longrightarrow M_{j} \\
& \left(m_{1}, \ldots, m_{j-1}, m_{j+1}, \ldots, m_{N_{E}}\right) \longrightarrow S\left(m_{1}, \ldots, m_{j-1}, m_{j+1}, \ldots, m_{N_{E}}\right)=m_{j}^{*} \text {. }
\end{aligned}
$$

Lemma 4.3. The mapping $S_{j}$ is continuous if the weak $\left(L^{\infty}(0, T)\right)^{N_{E}-1}$ topology is taken in $\prod_{\substack{i=1 \\ i \neq j}}^{N_{E}} M_{i}$ and the weak $L^{\infty}(0, T)$ topology is taken in $M_{j}$.

Proof. In order to alleviate notation and without loss of generality, we write the proof for the case $j=1$.

Let $\left\{\left(m_{2}^{n}, \ldots, m_{N_{E}}^{n}\right)\right\} \subset \prod_{i=2}^{N_{E}} M_{i}$ and $\left(m_{2}, \ldots, m_{N_{E}}\right) \in \prod_{i=2}^{N_{E}} M_{i}$ be such that

$$
\left\{\left(m_{2}^{n}, \ldots, m_{N_{E}}^{n}\right)\right\} \rightarrow\left(m_{2}, \ldots, m_{N_{E}}\right) \quad * \text { weakly in }\left(L^{\infty}(0, T)\right)^{N_{E}-1} .
$$

Let $m_{1}^{n}=S_{1}\left(m_{2}^{n}, \ldots, m_{N_{E}}^{n}\right)$. Since $\left\{m_{1}^{n}\right\} \subset M_{1}$ is bounded in $L^{\infty}(0, T)$, there exists a subsequence $\left\{m_{1}^{n^{\prime}}\right\}$ converging *weakly in $L^{\infty}(0, T)$ to some element $m_{1} \in M_{1}$ (because $M_{1}$ is closed for this topology).

The proof will be finished if we show that $m_{1}=S_{1}\left(m_{2}, \ldots, m_{N_{E}}\right)$. To do this, it suffices to prove that

$$
J_{1}\left(m_{1}, m_{2}, \ldots, m_{N_{E}}\right) \leq J_{1}\left(\hat{m}_{1}, m_{2}, \ldots, m_{N_{E}}\right) \quad \forall \hat{m}_{1} \in M_{1}
$$

Clearly $\left\{\left(m_{1}^{n^{\prime}}, m_{2}^{n^{\prime}} \ldots, m_{N_{E}}^{n^{\prime}}\right)\right\} \rightarrow\left(m_{1}, m_{2}, \ldots, m_{N_{E}}\right) *$ weakly in $\left(L^{\infty}(0, T)\right)^{N_{E}}$. Using Lemma 4.1, the continuity of the functional $H_{1}$, the weak lower semicontinuity of the functional $\Theta_{1}$ and the decomposition (3.7), we deduce that

$$
\begin{aligned}
J_{1}\left(m_{1}, m_{2}, \ldots, m_{N_{E}}\right) & =\Theta_{1}\left(m_{1}\right)+H_{1}\left(\varrho\left(m_{1}, m_{2}, \ldots, m_{N_{E}}, \rho_{0}\right)\right) \\
\leq & \liminf _{n^{\prime} \rightarrow \infty} \Theta_{1}\left(m_{1}^{n^{\prime}}\right)+\lim _{n^{\prime} \rightarrow \infty} H_{1}\left(\varrho\left(m_{1}^{n^{\prime}}, m_{2}^{n^{\prime}}, \ldots, m_{N_{E}}^{n^{\prime}}, \rho_{0}\right)\right) \\
& =\liminf _{n^{\prime} \rightarrow \infty} J_{1}\left(m_{1}^{n^{\prime}}, m_{2}^{n^{\prime}}, \ldots, m_{N_{E}}^{n^{\prime}}\right) .
\end{aligned}
$$

Since $m_{1}^{n^{\prime}}=S_{1}\left(m_{2}^{n^{\prime}}, \ldots, m_{N_{E}}^{n^{\prime}}\right)$, it follows from the definition of the mapping $S_{1}$ that

$$
J_{1}\left(m_{1}^{n^{\prime}}, m_{2}^{n^{\prime}}, \ldots, m_{N_{E}}^{n^{\prime}}\right) \leq J_{1}\left(\hat{m}_{1}, m_{2}^{n^{\prime}}, \ldots, m_{N_{E}}^{n^{\prime}}\right) \quad \forall \hat{m}_{1} \in M_{1}
$$


Taking the inferior limit and using again the decomposition (3.7), Lemma 4.1, and the continuity of $H_{1}$, we obtain that for all $\hat{m}_{1} \in M_{1}$

$$
\begin{aligned}
\liminf _{n^{\prime} \rightarrow \infty} J_{1}\left(m_{1}^{n^{\prime}}, m_{2}^{n^{\prime}}, \ldots, m_{N_{E}}^{n^{\prime}}\right) & \leq \liminf _{n^{\prime} \rightarrow \infty} J_{1}\left(\hat{m}_{1}, m_{2}^{n^{\prime}}, \ldots, m_{N_{E}}^{n^{\prime}}\right) \\
& =\Theta_{1}\left(\hat{m}_{1}\right)+\lim _{n^{\prime} \rightarrow \infty} H_{1}\left(\varrho\left(\hat{m}_{1}, m_{2}^{n^{\prime}}, \ldots, m_{N_{E}}^{n^{\prime}}, \rho_{0}\right)\right) \\
& =\Theta_{1}\left(\hat{m}_{1}\right)+H_{1}\left(\varrho\left(\hat{m}_{1}, m_{2}, \ldots, m_{N_{E}}, \rho_{0}\right)\right) \\
& =J_{1}\left(\hat{m}_{1}, m_{2}, \ldots, m_{N_{E}}\right) .
\end{aligned}
$$

Therefore equation (4.7) holds.

Theorem 4.4. If $f_{j} \in C\left[\underline{m}_{j}, \bar{m}_{j}\right]$ and $f_{j}$ is strictly convex in $\left[\underline{m}_{j}, \bar{m}_{j}\right]$ for all $j=1, \ldots, N_{E}$, then the problem $(\mathcal{P})$ has at least a Nash equilibrium.

Proof. Let $S: \prod_{j=1}^{N_{E}} M_{j} \longrightarrow \prod_{j=1}^{N_{E}} M_{j}$ be the mapping defined by

$$
S\left(m_{1}, m_{2}, \ldots, m_{N_{E}}\right)=\left(S_{1}\left(m_{2}, m_{3}, \ldots, m_{N_{E}}\right), S_{2}\left(m_{1}, m_{3}, \ldots, m_{N_{E}}\right), \ldots, S_{N_{E}}\left(m_{1}, m_{2}, \ldots, m_{N_{E}-1}\right)\right) .
$$

Due to the definition of the mappings $S_{i}$, every fixed point of $S$ is a Nash equilibrium. The existence of a fixed point of $S$ is ensured by second Schauder fixed point theorem [16], since the set $\prod_{j=1}^{N_{E}} M_{j}$ is convex and compact for the weak* $\left(L^{\infty}(0, T)\right)^{N_{E}}$ topology, and the mapping $S$ is continuous for this topology.

\section{Optimality CONDitions}

Since $M_{j}$ is convex, from Theorem 3.4, a necessary and sufficient condition to achieve (2.4) is

$$
\frac{\partial J_{i}}{\partial m_{i}}\left(m_{1}^{*}, \ldots, m_{i-1}^{*}, m_{i}^{*}, m_{i+1}^{*}, \ldots, m_{N_{E}}^{*}\right)\left(m_{i}-m_{i}^{*}\right) \geq 0, \forall m_{i} \in M_{i} .
$$

Then, our goal consists of finding $\left(m_{1}^{*}, \ldots, m_{N_{E}}^{*}\right) \in \prod_{j=1}^{N_{E}} M_{j}$ satisfying the following optimality system:

$$
\begin{aligned}
& \frac{\partial J_{1}}{\partial m_{1}}\left(m_{1}^{*}, \ldots, m_{i-1}^{*}, m_{i}^{*}, m_{i+1}^{*}, \ldots, m_{N_{E}}^{*}\right)\left(m_{1}-m_{1}^{*}\right) \geq 0, \quad \forall m_{1} \in M_{1} \\
& \frac{\partial J_{2}}{\partial m_{2}}\left(m_{1}^{*}, \ldots, m_{i-1}^{*}, m_{i}^{*}, m_{i+1}^{*}, \ldots, m_{N_{E}}^{*}\right)\left(m_{2}-m_{2}^{*}\right) \geq 0, \quad \forall m_{2} \in M_{2} \\
& \frac{\partial J_{N_{E}}}{\partial m_{N_{E}}}\left(m_{1}^{*}, \ldots, m_{i-1}^{*}, m_{i}^{*}, m_{i+1}^{*}, \ldots, m_{N_{E}}^{*}\right)\left(m_{N_{E}}-m_{N_{E}}^{*}\right) \geq 0, \quad \forall m_{N_{E}} \in M_{N_{E}}
\end{aligned}
$$

In order to simplify this system, we are going to obtain $\frac{\partial J_{j}}{\partial m_{j}}(m)$, for all $m=\left(m_{1}, m_{2}, \ldots, m_{N_{E}}\right) \in \prod_{j=1}^{N_{E}} M_{j}$. For the purpose of illustrating the process, in a similar way as described in [15], we begin to make calculations in a "formal" way. Denoting by $\delta_{j} m_{j}$ a small perturbation of $m_{j}$, we have

$$
\begin{aligned}
\delta_{j} J_{j}(m) & =\int_{0}^{T} \frac{\partial J_{j}}{\partial m_{j}}(m) \delta_{j} m_{j} \mathrm{~d} t \\
& =\int_{0}^{T} f^{\prime}\left(m_{j}\right) \delta_{j} m_{j} \mathrm{~d} t+\sum_{i=n_{(j-1)}+1}^{n_{j}} \frac{1}{\epsilon_{i}} \int_{\bar{A}_{i} \times(0, T)} \psi^{\prime}\left(\rho(x, t)-\sigma_{i}\right) \delta_{j} \rho \mathrm{d} x \mathrm{~d} t,
\end{aligned}
$$


where $\delta_{j} \rho(x, t)$ is the solution of

$$
\begin{array}{rc}
\frac{\partial \delta_{j} \rho}{\partial t}+\vec{u} \cdot \nabla \delta_{j} \rho-\beta \Delta \delta_{j} \rho+\kappa \delta_{j} \rho=\frac{1}{h} \delta_{j} m_{j} \delta\left(x-P_{j}\right) & \text { in } \Omega \times(0, T) \\
\delta_{j} \rho(x, 0)=0 & \text { in } \Omega \\
\frac{\partial \delta_{j} \rho}{\partial n}=0 & \text { on } \partial \Omega \times(0, T) .
\end{array}
$$

Let us introduce a reasonably smooth function $q_{j}(x, t)$ defined over $\bar{\Omega} \times[0, T]$. Multiplying equation (5.3) by $q_{j}(x, t)$ and integrating over $\Omega \times(0, T)$, we obtain

$$
\int_{\Omega \times(0, T)} q_{j}\left[\frac{\partial \delta_{j} \rho}{\partial t}+\vec{u} \cdot \nabla \delta_{j} \rho-\beta \Delta \delta_{j} \rho+\kappa \delta_{j} \rho\right] \mathrm{d} x \mathrm{~d} t=\int_{0}^{T} \frac{1}{h\left(P_{j}, t\right)} q_{j}\left(P_{j}, t\right)\left(\delta_{j} m_{j}\right)(t) \mathrm{d} t .
$$

Taking into account boundary and initial conditions (5.4) and (5.5), using Green's formula and integration by parts, we have

$$
\begin{aligned}
& \int_{\Omega \times(0, T)}\left(-\frac{\partial q_{j}}{\partial t}-\operatorname{div}\left(q_{j} \vec{u}\right)-\beta \Delta q_{j}+\kappa q_{j}\right) \delta_{j} \rho \mathrm{d} x \mathrm{~d} t \\
& \quad+\int_{\Omega} q_{j}(x, T) \delta_{j} \rho(x, T) \mathrm{d} x+\int_{\partial \Omega \times(0, T)}\left(\beta \frac{\partial q_{j}}{\partial n}+q_{j} \vec{u} \cdot \vec{n}\right)\left(\delta_{j} \rho\right) \mathrm{d} \Gamma \mathrm{d} t=\int_{0}^{T} \frac{1}{h\left(P_{j}, t\right)} q_{j}\left(P_{j}, t\right)\left(\delta_{j} m_{j}\right)(t) \mathrm{d} t .
\end{aligned}
$$

Now, in order to simplify the expression of $\frac{\partial J_{j}}{\partial m_{j}}(m)$, we choose $q_{j}$ as the solution, in the sense of Definition 5.1, of the following adjoint problem:

$$
\begin{aligned}
-\frac{\partial q_{j}}{\partial t}-\beta \Delta q_{j}-\operatorname{div}\left(q_{j} \vec{u}\right)+\kappa q_{j} & =\sum_{i=n_{(j-1)}+1}^{n_{j}} \frac{1}{\epsilon_{i}} \chi_{\bar{A}_{i}} \psi^{\prime}\left(\rho-\sigma_{i}\right) \quad \text { in } \Omega \times(0, T) \\
\beta \frac{\partial q_{j}}{\partial n}+q_{j}(\vec{u} \cdot \vec{n}) & =0 \text { on } \partial \Omega \times(0, T) \\
q_{j}(x, T) & =0 \text { in } \Omega,
\end{aligned}
$$

where $\chi_{\bar{A}_{i}}$ denotes the characteristic function of the set $\bar{A}_{i}$.

Definition 5.1. We say that $q_{j} \in L^{2}\left(0, T ; H^{1}(\Omega)\right) \cap C\left([0, T] ; L^{2}(\Omega)\right)$ is a solution of $(5.8)-(5.10)$ if $q_{j}(x, T)=0$ a.e. in $\Omega$ and verifies

$$
\begin{aligned}
-\frac{\mathrm{d}}{\mathrm{d} t} \int_{\Omega} q_{j}(x, t) v(x) & \mathrm{d} x+\beta \int_{\Omega} \nabla q_{j}(x, t) \cdot \nabla v(x) \mathrm{d} x+\int_{\Omega} q_{j}(x, t) \vec{u} \cdot \nabla v(x) \mathrm{d} x \\
+\int_{\Omega} \kappa q_{j}(x, t) v(x) \mathrm{d} x & =\sum_{i=n_{(j-1)}+1}^{n_{j}} \frac{1}{\epsilon_{i}} \int_{\Omega} \chi_{\bar{A}_{i}}(x) \psi^{\prime}\left(\rho(x, t)-\sigma_{i}\right) v(x) \mathrm{d} x \quad \forall v \in H^{1}(\Omega)
\end{aligned}
$$

in the sense of $\mathcal{D}^{\prime}((0, T))$. 
The following result arises from Section 9 of Chapter IV of [9] (Th. 9.1 concerns the regularity of solution for a Dirichlet boundary value problem, but it is also valid in our case - with a Robin boundary condition - as it has stated in the last paragraph of that section).

Theorem 5.2. The problem (5.8)-(5.10) has only one solution $q_{j}$. Moreover $q_{j} \in L^{2}\left(0, T ; H^{2}(\Omega)\right) \cap H^{1}(0, T$; $\left.L^{2}(\Omega)\right)$.

Taking $q_{j}$ as the solution of (5.8)-(5.10), equation (5.7) becomes

$$
\sum_{i=n_{(j-1)}+1}^{n_{j}} \frac{1}{\epsilon_{i}} \int_{\bar{A}_{i} \times(0, T)} \psi^{\prime}\left(\rho(x, t)-\sigma_{i}\right) \delta_{j} \rho \mathrm{d} x \mathrm{~d} t=\int_{0}^{T} \frac{1}{h\left(P_{j}, t\right)}\left(\delta_{1} m_{j}\right)(t) q_{j}\left(P_{j}, t\right) \mathrm{d} t .
$$

Now, if (5.12) is substituted in the initial expression (5.2) we obtain

$$
\int_{0}^{T} \frac{\partial J_{j}}{\partial m_{j}}(m) \delta_{j} m_{j} \mathrm{~d} t=\int_{0}^{T} f_{j}^{\prime}\left(m_{j}\right) \delta_{j} m_{j} \mathrm{~d} t+\int_{0}^{T} \frac{1}{h\left(P_{j}, t\right)}\left(\delta_{j} m_{j}\right)(t) q_{j}\left(P_{j}, t\right) \mathrm{d} t,
$$

consequently

$$
\frac{\partial J_{j}}{\partial m_{j}}(m)=f_{j}^{\prime}\left(m_{j}\right)+\frac{1}{h\left(P_{j}, t\right)} q_{j}\left(P_{j}, t\right)
$$

Remark 5.3. Although the previous calculations have been made in a "formal" way, the regularity given by Theorems 3.2 and 5.2 for functions $\delta_{j} \rho$ (solution of (5.3)-(5.5)) and $q_{j}$ (solution of (5.8)-(5.10)), is sufficient to prove that (5.13) is strictly correct.

Taking the expression (5.13) in the system (5.1), we can state the following result:

Theorem 5.4. The element $\left(m_{1}^{*}, m_{2}^{*}, \ldots, m_{N_{E}}^{*}\right) \in \prod_{j=1}^{N_{E}} M_{j}$ is a Nash equilibrium of the problem $(\mathcal{P})$ if and only if there exists one function $\rho \in L^{r}\left(0, T ; W^{1, s}(\Omega)\right) \cap L^{2}\left(0, T ; L^{2}(\Omega)\right)$ for all $r, s \in[1,2)$ with $\frac{2}{r}+\frac{2}{s}>3$ and $N_{E}$ functions $q_{1}, q_{2}, \ldots, q_{N_{E}} \in C\left([0, T] ; L^{2}(\Omega)\right) \cap L^{2}\left([0, T] ; H^{1}(\Omega)\right)$, such that: for $k=1,2, \ldots, N_{E}$,

$$
\begin{array}{rc}
\frac{\partial \rho}{\partial t}+\vec{u} \cdot \nabla \rho-\beta \Delta \rho+\kappa \rho=\sum_{j=1}^{N_{E}} \frac{1}{h}\left[m_{j}^{*} \delta\left(x-P_{j}\right)\right] & \text { in } \Omega \times(0, T) \\
\rho(x, 0)=\rho_{0}(x) & \text { in } \Omega \\
\frac{\partial \rho}{\partial n}=0 & \text { on } \partial \Omega \times(0, T) \\
-\frac{\partial q_{k}}{\partial t}-\beta \Delta q_{k}-\operatorname{div}\left(q_{k} \vec{u}\right)+\kappa q_{k}=\sum_{i=n_{(k-1)}+1}^{n_{k}} \frac{1}{\epsilon_{i}} \chi_{\bar{A}_{i}} \psi^{\prime}\left(\rho-\sigma_{i}\right) & \text { in } \Omega \times(0, T) \\
\beta \frac{\partial q_{k}}{\partial n}+q_{k}(\vec{u} \cdot \vec{n})=0 & \text { on } \partial \Omega \times(0, T) \\
q_{k}(x, T)=0 & \text { in } \Omega \\
\int_{0}^{T}\left[f_{k}^{\prime}\left(m_{k}^{*}\right)+\frac{1}{h\left(P_{k}, t\right)} q_{k}\left(P_{k}, t\right)\right]\left(m_{k}-m_{k}^{*}\right) \mathrm{d} t \geq 0 & \forall m_{k} \in M_{k} .
\end{array}
$$




\section{Numerical SOLUTiON}

In order to simplify notations and without loss of generality, we shall assume in the sequel $N_{E}=2$ and $N_{Z}=2$, with $n_{1}=1$ and $n_{2}=2$. Moreover, if we suppose that penalization on CF concentration is nontrivial (some purification is necessary to avoid CF concentration greater than threshold levels in protected areas) and $\underline{m}_{j}$ is sufficiently close to zero, we can assume that the minimum in (2.4) is attained at an interior point of $M_{j}$ because of the particular form of the function $f_{j}$ (see Fig. 2). In this case, the optimality system (5.14)-(5.20) reads: for $k=1,2$,

$$
\begin{array}{rc}
\frac{\partial \rho}{\partial t}+\vec{u} \cdot \nabla \rho-\beta \Delta \rho+\kappa \rho=\frac{1}{h}\left[m_{1}^{*} \delta\left(x-P_{1}\right)+m_{2}^{*} \delta\left(x-P_{2}\right)\right] & \text { in } \Omega \times(0, T) \\
\rho(x, 0)=\rho_{0}(x) & \text { in } \Omega \\
\frac{\partial \rho}{\partial n}=0 & \text { on } \partial \Omega \times(0, T) \\
-\frac{\partial q_{k}}{\partial t}-\beta \Delta q_{k}-\operatorname{div}\left(q_{k} \vec{u}\right)+\kappa q_{k}=\frac{1}{\epsilon_{k}} \chi_{\bar{A}_{k}} \psi^{\prime}\left(\rho-\sigma_{k}\right) & \text { in } \Omega \times(0, T) \\
\beta \frac{\partial q_{k}}{\partial n}+q_{k}(\vec{u} \cdot \vec{n})=0 & \text { on } \partial \Omega \times(0, T) \\
q_{k}(x, T)=0 & \text { in } \Omega \\
f_{k}^{\prime}\left(m_{k}^{*}\right)+\frac{1}{h\left(P_{k}, t\right)} q_{k}\left(P_{k}, t\right)=0 & \text { in }(0, T) .
\end{array}
$$

In this section, we shall deal with the numerical solution of this system.

\subsection{Discretization of the system}

Solving the preceding system needs, as previous steps, to be able to solve the state system (6.1)-(6.3) and the adjoint state system (6.4)-(6.6). To do this, we shall use a method which combines a time discretization by characteristics with an space discretization by Lagrange $P_{1}$ finite elements. This method is convergent for the adjoint system (see [14]) and also for the state system (see [1]).

We begin by making a time discretization in which the convective terms in (6.1) and (6.4) are treated with the characteristics method ( $c f$. [14]). This method is based upon the equality

$$
\frac{D y}{D t}(x, t)=\frac{\partial y}{\partial t}(x, t)+\vec{u} \cdot \nabla y
$$

where $\frac{D y}{D t}$ stands for the material derivative of $y$ with respect to $\vec{u}$ and $t$, that is

$$
\frac{D y}{D t}(x, t)=\left.\frac{\partial}{\partial \tau}[y(X(x, t ; \tau), \tau)]\right|_{\tau=t}
$$

Here $\tau \rightarrow X(x, t ; \tau)$ is the trajectory which follows the particle of fluid being at point $x$ at time $t$. This function, named characteristic, can be obtained as the solution of the following initial value problem

$$
\begin{aligned}
\frac{\mathrm{d} X}{\mathrm{~d} \tau} & =\vec{u}(X(x, t ; \tau), \tau) \\
X(x, t ; t) & =x .
\end{aligned}
$$


We take $N \in \mathbb{N}, \Delta t=\frac{T}{N}$ and we define $t_{n}=n \Delta t$. We denote by $X^{n}(x)=X\left(x, t^{n+1} ; t^{n}\right)$ the position at instant $t^{n}$ of the particle that will be at point $x$ in the instant $t^{n+1}$ and by $Y^{n+1}(x)=X\left(x, t^{n} ; t^{n+1}\right)$ the position at instant $t^{n+1}$ of the particle that is at point $x$ in the instant $t^{n}$. Then we make the approximations

$$
\begin{gathered}
\frac{\partial \rho}{\partial t}+\vec{u} \cdot \nabla \rho=\frac{D \rho}{D t} \simeq \frac{\rho^{n+1}(x)-\rho^{n}\left(X^{n}(x)\right)}{\Delta t}, \\
-\frac{\partial q_{k}}{\partial t}-\vec{u} \cdot \nabla q_{k}=-\frac{D q_{k}}{D t} \simeq \frac{q_{k}^{n}(x)-q_{k}^{n+1}\left(Y^{n+1}(x)\right)}{\Delta t}, \quad k=1,2 .
\end{gathered}
$$

This leads us to approximate the system (6.1)-(6.7) by the following semidiscrete problem: for $k=1,2$, $n=0,1, \ldots, N-1$,

$$
\begin{array}{rr}
\frac{\rho^{n+1}-\rho^{n} \circ X^{n}}{\Delta t}-\beta \Delta \rho^{n+1}+\kappa \rho^{n+1}=\frac{1}{h^{n+1}}\left[m_{1}^{n+1} \delta\left(x-P_{1}\right)+m_{2}^{n+1} \delta\left(x-P_{2}\right)\right] & \text { in } \Omega \\
\frac{\partial \rho^{n+1}}{\partial n}=0 & \text { on } \partial \Omega \\
\frac{q_{k}^{n}(x)-q_{k}^{n+1} \circ Y^{n+1}}{\Delta t}-q_{k}^{n} \operatorname{div} \vec{u}^{n}-\beta \Delta q_{k}^{n}+\kappa q_{k}^{n}=\frac{1}{\epsilon_{k}} \chi_{\bar{A}_{k}} \psi^{\prime}\left(\rho^{n}-\sigma_{k}\right) & \text { in } \Omega \\
\beta \frac{\partial q_{k}^{n}}{\partial n}+q_{k}^{n}\left(\vec{u}^{n} \cdot \vec{n}\right)=0 & \text { on } \partial \Omega \\
f_{k}^{\prime}\left(m_{k}^{n+1}\right)+\frac{1}{h^{n+1}\left(P_{k}\right)} q_{k}^{n+1}\left(P_{k}\right)=0, &
\end{array}
$$

where $\rho^{0}=\rho_{0}, q_{1}^{N}=q_{2}^{N}=0, \vec{u}^{n}=\vec{u}\left(., t_{n}\right), h^{n}=\vec{h}\left(., t_{n}\right)$ and $m_{k}^{n}=m_{k}^{*}\left(t_{n}\right)$.

Now we approximate $\Omega$ by the polygonal set $\Omega_{h}$ and choose an admissible triangulation $\tau_{h}$ of it with triangles of diameter equal or less than $h$, such that the vertices on the boundary of $\Omega_{h}$ also lie on the boundary of $\Omega$. Let $\left\{x_{j}, j=1, \ldots, N_{v}\right\}$ be the set of the vertices of $\tau_{h}$. For each $n=0,1, \ldots, N-1$ we consider the variational formulation of the problems (6.13)-(6.14) and (6.15)-(6.16). In spite of $\rho$ could be not continuous near the points $P_{1}, P_{2}, \ldots, P_{N_{E}}$, we know that $\rho \in L^{r}\left(0, T ; W^{1, s}(\Omega)\right)$. Then, it seems reasonable to look for the fully discrete solution $\rho_{h}^{n}$ in a space $V_{h}$ that would be an internal approximation of $W^{1, s}\left(\Omega_{h}\right)$. Taking into account that any piecewise polynomial function in $W^{1, s}\left(\Omega_{h}\right)$ is continuous, we take $V_{h}=\left\{v_{h} \in C\left(\bar{\Omega}_{h}\right),\left.v_{h}\right|_{T} \in P_{1}, T \in \tau_{h}\right\}$ (note that $V_{h}=\left\{v_{h} \in W^{1, s}\left(\Omega_{h}\right),\left.v_{h}\right|_{T} \in P_{1}, T \in \tau_{h}\right\}$ ). Moreover, this choice provides good convergence properties (cf. [1] and [14]).

Let $\rho_{h}^{0} \in V_{h}$ be an approximation of $\rho^{0}$. We set $q_{1 h}^{N}=q_{2 h}^{N}=0$ and we denote by $X_{h}^{n}$ and $Y_{h}^{n+1}$ the approximations of $X^{n}$ and $Y^{n+1}$ obtained by solving the system (6.10) with the backward Euler scheme. Thus, system (6.13)-(6.14) is discretized by the following problem: find $\rho_{h}^{n+1} \in V_{h}$ satisfying

$$
\begin{aligned}
\int_{\Omega} \frac{\rho_{h}^{n+1}-\rho_{h}^{n} \circ X_{h}^{n}}{\Delta t} v_{h}+\beta \int_{\Omega} \nabla \rho_{h}^{n+1} \cdot \nabla v_{h}+\kappa \int_{\Omega} \rho_{h}^{n+1} v_{h}= & \frac{m_{1}^{n+1}}{h^{n+1}\left(P_{1}\right)} v_{h}\left(P_{1}\right) \\
& +\frac{m_{2}^{n+1}}{h^{n+1}\left(P_{2}\right)} v_{h}\left(P_{2}\right), \quad \forall v_{h} \in V_{h} .
\end{aligned}
$$


In the same way, (6.15)-(6.16) is discretized by the following problem: find $q_{h}^{n} \in V_{h}$ satisfying

$$
\begin{array}{r}
\int_{\Omega} \frac{q_{k h}^{n}-q_{k h}^{n+1} \circ Y_{h}^{n+1}}{\Delta t} v_{h}+\int_{\Omega} q_{k h}^{n} \vec{u}^{n} \cdot \nabla v_{h}+\int_{\Omega} \vec{u}^{n} \cdot\left(\nabla q_{k h}^{n}\right) v_{h}+\beta \int_{\Omega} \nabla q_{k h}^{n} \cdot \nabla v_{h}+\kappa \int_{\Omega} q_{k h}^{n} v_{h}= \\
\frac{1}{\epsilon_{k}} \int_{\Omega} \chi_{\bar{A}_{k}} \psi^{\prime}\left(\rho_{h}^{n}-\sigma_{k}\right) v_{h}, \quad \forall v_{h} \in V_{h} .
\end{array}
$$

Let $\mathcal{B}_{V_{h}}=\left\{\tilde{v}_{1}, \tilde{v}_{2}, \ldots, \tilde{v}_{N_{v}}\right\}$ be the nodal basis of $V_{h}$, that is, $\tilde{v}_{i}\left(x_{j}\right)=\delta_{i j}$ for all $i, j=1,2, \ldots, N_{v}$. As it is well known, equations (6.18) and (6.19) can be written in a matrix form by using the nodal basis. In this way, the system (6.13)-(6.17) is approximated by the following fully discrete system:

Given $\hat{\rho}_{h}^{0}=\left(\rho_{h 0}\left(x_{1}\right), \rho_{h 0}\left(x_{2}\right), \ldots, \rho_{h 0}\left(x_{N_{v}}\right)\right)^{t}$ and $\hat{q}_{1 h}^{N}=\hat{q}_{2 h}^{N}=(0,0, \ldots, 0)^{t}$, for $k=1,2$ and $n=0,1, \ldots$, $N-1$, find $m_{k}^{n+1} \in \mathbb{R}, \hat{\rho}_{h}^{n+1} \in \mathbb{R}^{N_{v}}, \hat{q}_{k h}^{n} \in \mathbb{R}^{N_{v}}$ satisfying:

$$
\begin{aligned}
A_{1 h} \hat{\rho}_{h}^{n+1}-B_{1 h}^{n} \hat{\rho}_{h}^{n} & =\frac{m_{1}^{n+1}}{h^{n+1}\left(P_{1}\right)} b_{1 h}+\frac{m_{2}^{n+1}}{h^{n+1}\left(P_{2}\right)} b_{2 h}, \\
A_{2 h}^{n} \hat{q}_{k h}^{n}-B_{2 h}^{n+1} \hat{q}_{k h}^{n+1} & =\beta_{k h}^{n}, \\
f_{k}^{\prime}\left(m_{k}^{n+1}\right) & =-\frac{1}{h^{n+1}\left(P_{k}\right)} C_{k h} \hat{q}_{k h}^{n+1},
\end{aligned}
$$

where, for $i, j=1,2, \ldots, N_{v}$ :

- $\hat{\rho}_{h}^{n+1}=\left(\rho_{h}^{n+1}\left(x_{1}\right), \ldots, \rho_{h}^{n+1}\left(x_{N_{v}}\right)\right)^{t}, \hat{q}_{k h}^{n+1}=\left(q_{k h}^{n+1}\left(x_{1}\right), \ldots, q_{k h}^{n+1}\left(x_{N_{v}}\right)\right)^{t}$

- $\left(A_{1 h}\right)_{i j}=\left(\frac{1}{\Delta t}+\kappa\right) \int_{\Omega} \tilde{v}_{j} \tilde{v}_{i}+\beta \int_{\Omega} \nabla \tilde{v}_{j} \cdot \nabla \tilde{v}_{i}$

- $\left(B_{1 h}^{n}\right)_{i j}=\frac{1}{\Delta t} \int_{\Omega} \tilde{v}_{j}\left(X_{h}^{n}(x)\right) \tilde{v}_{i}$

- $\left(A_{2 h}^{n}\right)_{i j}=\left(\frac{1}{\Delta t}+\kappa\right) \int_{\Omega} \tilde{v}_{j} \tilde{v}_{i}+\beta \int_{\Omega} \nabla \tilde{v}_{j} \cdot \nabla \tilde{v}_{i}+\int_{\Omega} \tilde{v}_{j}\left(\vec{u}^{n} \cdot \nabla \tilde{v}_{i}\right)+\int_{\Omega}\left(\vec{u}^{n} \cdot \nabla \tilde{v}_{j}\right) \tilde{v}_{i}$

- $\left(B_{2 h}^{n+1}\right)_{i j}=\frac{1}{\Delta t} \int_{\Omega} \tilde{v}_{j}\left(Y_{h}^{n+1}(x)\right) \tilde{v}_{i}$

- $\left(b_{k h}\right)_{i}=\tilde{v}_{i}\left(P_{k}\right)$

- $\left(\beta_{k h}^{n}\right)_{i}=\frac{1}{\epsilon_{k}} \int_{\Omega} \chi_{\bar{A}_{k}} \psi^{\prime}\left(\sum_{l=1}^{N_{v}}\left(\hat{\rho}_{h}^{n}\right)_{l} \tilde{v}_{l}-\sigma_{k}\right) \tilde{v}_{i}$

- $C_{k h}$ is the matrix of order $1 \times N_{v}$ of the linear mapping which gives the value $q_{k h}^{n+1}\left(P_{k}\right)$ from the vector $\hat{q}_{k h}^{n+1}$.

These integrals are computed by quadrature formulae. In particular, to obtain $B_{1 h}^{n}$ and $B_{2 h}^{n+1}$, we compute previously the values of $X_{h}^{n}$ and $Y_{h}^{n+1}$ at quadrature points. In general, they are not mesh points but the value of $\tilde{v}_{j}$ at them can also be easily calculated.

System (6.20)-(6.22) is equivalent to a discretization of optimality system (6.1)-(6.7), but it is not the optimality system of the discrete problem. In effect, with the previous discretization we could write the discrete problem and then we could derive the corresponding optimality system. This alternative is also valid (with advantages and disadvantages) and will be studied in a forthcoming paper. 


\subsection{Solving the discrete system}

Since $\beta_{k h}^{n}$ depends on $\hat{\rho}_{h}^{n}$ in a nonlinear way, the discrete system (6.20)-(6.22) is fully coupled, square and nonlinear. At first glance, its dimension is $3 N N_{v}+2 N$.

Our goal is to compute the vector $\tilde{m}=\left(\tilde{m}_{1}, \tilde{m}_{2}\right)^{t} \in \mathbb{R}^{2 N}$, where $\tilde{m}_{k}=\left(m_{k}^{1}, \ldots, m_{k}^{N}\right)^{t}, k=1,2$. This vector gives us the values of the Nash equilibrium at the points of the time grid. We shall introduce a function $F: \mathbb{R}^{2 N} \rightarrow \mathbb{R}^{2 N}$ in such a way that $(6.20)-(6.22)$ be equivalent to $F(\tilde{m})=0$. This allows us to reduce the dimension of the system. In order to define this function, we assume that each of the protected zones $\bar{A}_{1}$ and $\bar{A}_{2}$ is formed by the union of some triangles of $\tau_{h}$. So we can define the triangulation $\tau_{z} \subset \tau_{h}$ as $\tau_{z}=\left\{T \in \tau_{z} ; \cup_{T \in \tau_{z}}=\bar{A}_{1} \cup \bar{A}_{2}\right\}$. Let $\left\{y_{i}, i=1, \ldots, N_{z_{1}}\right\}$ be the set of the vertices of $\tau_{z}$ lying in $\bar{A}_{1}$ and $\left\{y_{i}, i=N_{z_{1}}+1, \ldots, N_{z}\right\}$ that of the vertices in $\bar{A}_{2}$. Since any vertex of $\tau_{z}$ is a vertex of $\tau_{h}$, we have $y_{i}=x_{l(i)}, i=1, \ldots, N_{z}$, where $l$ is an injective mapping from $\left\{1, \ldots, N_{z}\right\}$ into $\left\{1, \ldots, N_{v}\right\}$.

We now consider the following functions:

(1) Function $G_{1}$

$$
G_{1}: \tilde{m} \in \mathbb{R}^{2 N} \rightarrow \tilde{c}_{h} \in \mathbb{R}^{N_{z}(N+1)}
$$

where $\tilde{c}_{h}=\left(\hat{c}_{h}^{0}, \hat{c}_{h}^{1}, \ldots, \hat{c}_{h}^{N}\right)^{t}$ is defined by

$$
\left(\hat{c}_{h}^{n}\right)_{i}=\left(\hat{\rho}_{h}^{n}\right)_{l(i)}, i=1, \ldots, N_{z}
$$

From the numerical point of view, mapping $G_{1}$ consists of solving (6.20) and extracting from the solution the components corresponding to the vertices of the triangulation lying on the protected zones. Since (6.20) is a linear problem, the mapping $G_{1}$ is affine. So the matrix $D G_{1} \in M_{N_{z}(N+1) \times 2 N}$ and the vector $G_{1}(0) \in \mathbb{R}^{N_{z}(N+1)}$ can be computed and stored once for all. Each time the vector $G_{1}(\tilde{m})$ is needed, it can be computed as

$$
G_{1}(\tilde{m})=D G_{1} \tilde{m}+G_{1}(0)
$$

(2) Function $G_{2}$

$$
G_{2}: \tilde{c_{h}} \in \mathbb{R}^{N_{z}(N+1)} \rightarrow \tilde{d}_{h} \in \mathbb{R}^{N_{z}(N+1)},
$$

with $\tilde{d}_{h}=\left(\hat{d}_{h}^{0}, \hat{d}_{h}^{1}, \ldots, \hat{d}_{h}^{N}\right)^{t}$ defined by

$$
\left(\hat{d}_{h}^{n}\right)_{i}= \begin{cases}\frac{1}{3 \epsilon_{1}} U_{i} \psi^{\prime}\left(\left(\hat{c}_{h}^{n}\right)_{i}-\sigma_{1}\right) & \text { if } 1 \leq i \leq N_{z_{1}} \\ \frac{1}{3 \epsilon_{2}} V_{i} \psi^{\prime}\left(\left(\hat{c}_{h}^{n}\right)_{i}-\sigma_{2}\right) & \text { if } N_{z_{1}}+1 \leq i \leq N_{z}\end{cases}
$$

where:

- $U_{i}$ is the sum of the areas of the triangles contained in $\bar{A}_{1}$ having $y_{i}$ as a vertex for $i=1,2, \ldots, N_{z_{1}}$;

- $V_{i}$ is the sum of the areas of the triangles contained in $\bar{A}_{2}$ having $y_{i}$ as a vertex for $i=N_{z_{1}}+$ $1, \ldots, N_{z}$. 
Function $G_{2}$ furnishes an approximation to the components of $\beta_{k h}^{n}$, for $k=1,2$ and $n=0,1, \ldots, N$, corresponding to the vertices lying on $\bar{A}_{1} \cup \bar{A}_{2}$. (More precisely, this approximation is obtained by using the quadrature formula

$$
\int_{T} \Psi(x) \mathrm{d} x \simeq \frac{1}{3} \operatorname{area}(T)\left(\Psi\left(a_{T, 1}\right)+\Psi\left(a_{T, 2}\right)+\Psi\left(a_{T, 3}\right)\right)
$$

for a generic triangle $T$ whose vertices are denoted $a_{T, 1}, a_{T, 2}$ and $a_{T, 3}$.) From the numerical point of view, once the vectors $\left(U_{1}, \ldots, U_{N_{z_{1}}}\right)$ and $\left(V_{N_{Z_{1}}+1}, \ldots, V_{N_{z}}\right)$ are known, the computation of $G_{2}\left(\tilde{c}_{h}\right)$ and the Jacobian matrix $D G_{2}\left(\tilde{c}_{h}\right)$ can be carried out independently of the grid $\tau_{h}$. Moreover, for each $\tilde{c}_{h} \in \mathbb{R}^{N_{z}(N+1)}$, the matrix $D G_{2}\left(\tilde{c}_{h}\right) \in M_{N_{z}(N+1) \times N_{z}(N+1)}$ is diagonal and is given by

$$
\begin{aligned}
& \left(D G_{2}\left(\tilde{c}_{h}\right)\right)_{(n-1) N_{Z}+i,(n-1) N_{Z}+i}= \begin{cases}\frac{1}{3 \epsilon_{1}} U_{i} \psi^{\prime \prime}\left(\left(\hat{c}_{h}^{n}\right)_{i}-\sigma_{1}\right) & \text { if } 1 \leq i \leq N_{z_{1}} \\
\frac{1}{3 \epsilon_{2}} V_{i} \psi^{\prime \prime}\left(\left(\hat{c}_{h}^{n}\right)_{i}-\sigma_{2}\right) & \text { if } N_{z_{1}}+1 \leq i \leq N_{z}\end{cases} \\
& \text { for } n=1,2, \ldots, N+1 .
\end{aligned}
$$

(3) Function $G_{3}$

$$
G_{3}: \tilde{d}_{h} \in \mathbb{R}^{N_{z}(N+1)} \rightarrow G_{3}\left(\tilde{d}_{h}\right) \in \mathbb{R}^{2 N}
$$

defined by

$$
G_{3}\left(\tilde{d}_{h}\right)=\left[\left[\frac{1}{h^{n+1}\left(P_{k}\right)} C_{k h} \hat{q}_{k h}^{n+1}\right]_{n=0}^{N-1}\right]_{k=1}^{2}
$$

where $\hat{q}_{k h}^{n+1}$ is the solution of (6.21) with right hand side

$$
\begin{gathered}
\left(\beta_{1 h}^{n}\right)_{i}=\left\{\begin{array}{cl}
\left(\hat{d}_{h}^{n}\right)_{j} & \text { if } i=l(j), j=1, \ldots, N_{z_{1}} \\
0 & \text { otherwise }
\end{array}\right. \\
\left(\beta_{2 h}^{n}\right)_{i}=\left\{\begin{array}{cl}
\left(\hat{d}_{h}^{n}\right)_{j} & \text { if } i=l(j), j=N_{z_{1}}+1, \ldots, N_{z} \\
0 & \text { otherwise. }
\end{array}\right.
\end{gathered}
$$

From the numerical point of view, it is worthwhile to remark that $G_{3}$ is a linear mapping. So the matrix $D G_{3} \in M_{2 N \times N_{z}(N+1)}$ can be computed and stored once for all. Each time the vector $G_{3}\left(\tilde{d}_{h}\right)$ is needed, it can be computed as

$$
G_{3}\left(\tilde{d}_{h}\right)=D G_{3} \tilde{d}_{h} .
$$

Now we can define $F: \tilde{m} \in \mathbb{R}^{2 N} \rightarrow F(\tilde{m}) \in \mathbb{R}^{2 N}$ by

$$
F(\tilde{m})=G_{3}\left(G_{2}\left(G_{1}(\tilde{m})\right)\right)+\left[\left[f_{k}^{\prime}\left(m_{k}^{n+1}\right)\right]_{n=0}^{N-1}\right]_{k=1}^{2} .
$$

In this way, the optimality system (6.20)-(6.22) is equivalent to $F(\tilde{m})=0$. As we have quoted above, once the matrices $D G_{1}$ and $D G_{3}$ and the vector $G_{1}(0)$ have been computed, the computation of $F(\tilde{m})$ and $D F(\tilde{m})$ can be carried out independently of the grid and it is easy to perform. So we have solved numerically the system $F(\tilde{m})=0$ by using a Newton-like method. 


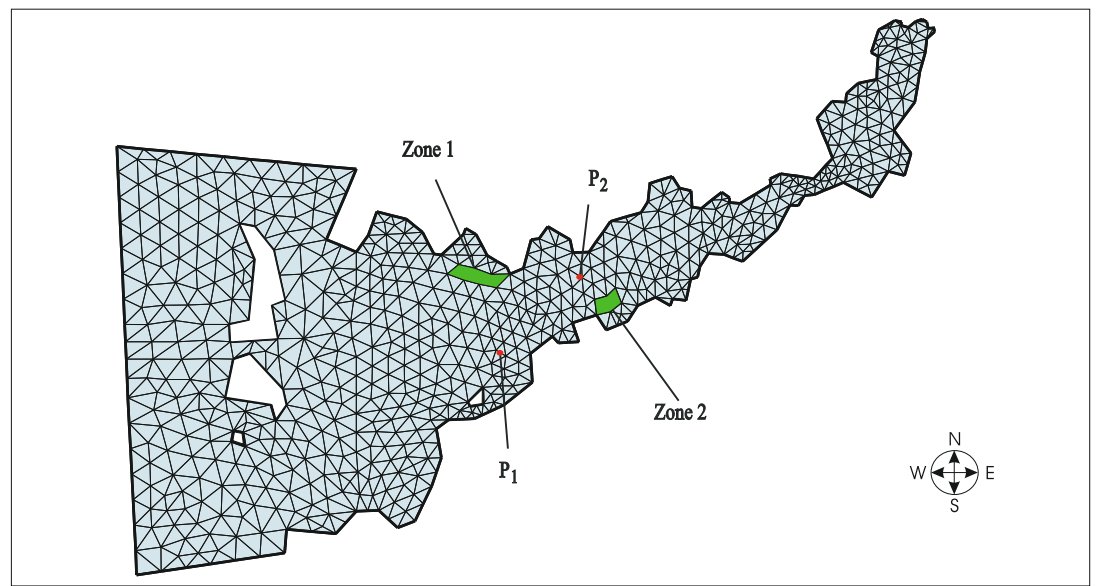

Figure 3. Grid of Vigo's estuary (Spain) used in the simulation.

\section{NUMERICAL RESULTS}

In this section we present the numerical results obtained by solving the previous problem in a realistic situation posed in the estuary of Vigo (Spain). The chosen grid and the location of discharged points and protected areas can be seen in Figure 3. For the simulation we consider a complete tidal cycle $(T=12.4 \mathrm{~h}$ in this latitude) and take the height and the velocity field obtained by solving the shallow water equations on the same grid (a detailed description of these equations can be seen, for instance, in [6]). Moreover, we have supposed null initial concentration of $\mathrm{CF}\left(\rho_{0}=0\right)$ and we have considered different thresholds for $\mathrm{CF}$ in each protected area $\left(\sigma_{1}=0.0003484, \sigma_{2}=0.0005\right)$. Finally, the same purification cost function has been taken for both plants:

$$
f_{1}(x)=f_{2}(x)= \begin{cases}\frac{100(150)^{3}}{x^{3}-3(150) x^{2}+3(150)^{2} x}, & x \leq 150 \\ 100, & x>150\end{cases}
$$

In order to observe the effect of penalization, we have carried out different experiments for different values of $\epsilon_{1}$ and $\epsilon_{2}$ :

(1) First, we take $\epsilon_{1}=\epsilon_{2}$ and we decrease its value to observe how the plants change their strategy of purification. In Figures 4 to 9 we can see, for different penalizations, the optimal discharges at each point (Figs. 4, 6 and 8) and the CF concentrations, corresponding to these discharges, in the neighborhood of protected areas at final time of simulation (Figs. 5, 7 and 9). As it is expected, we observe that bigger penalization (low values of $\epsilon_{1}=\epsilon_{2}$ ) corresponds with less discharges and, consequently, with less CF concentration.

(2) In the second experiment we have taken different penalizations for each plant. In contrast to the previous case, we observe that, when the penalization is smaller in one plant (see Figs. 10 and 11), the plant with more penalization (plant 2) must follow stronger strategy of purification (the discharges must be smaller). The stronger penalization of plant 2 provokes that the other plant can carry out slightly higher discharges than in the previous experiment (see Fig. 6 - same penalization - and $10-$ penalization stronger in plant 2). 


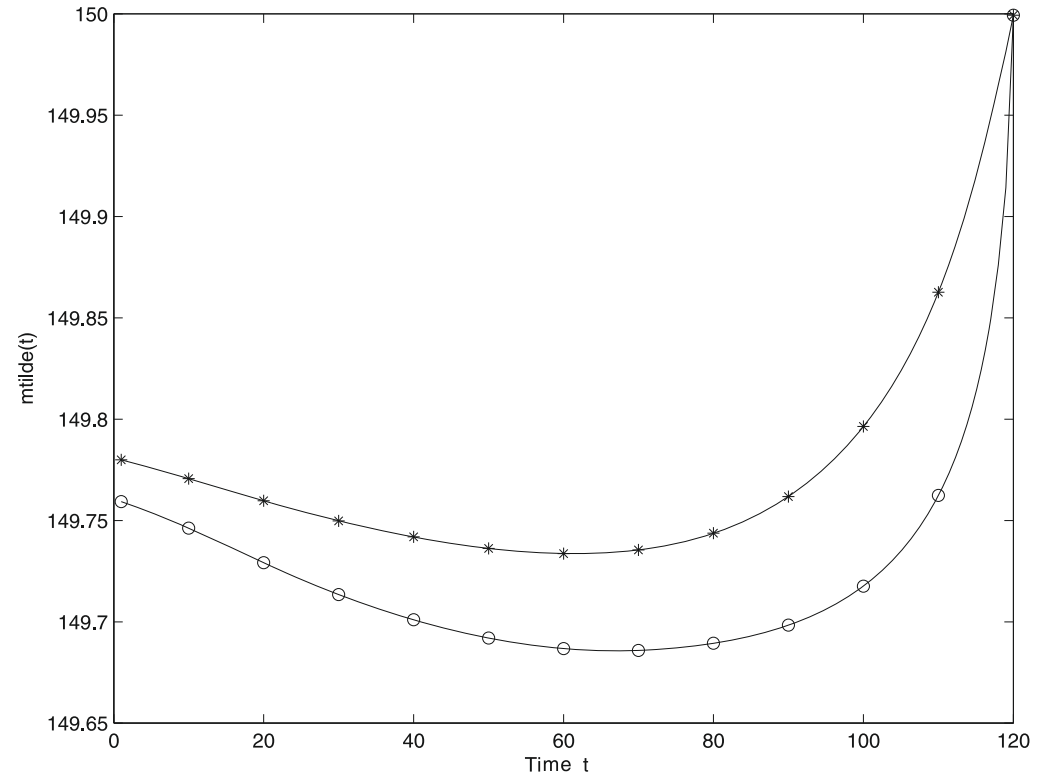

Figure 4. Optimal discharges $m_{1}^{*}(t)(* *)$ and $m_{2}^{*}(t)(o o)$ for $\epsilon_{1}=\epsilon_{2}=1$.

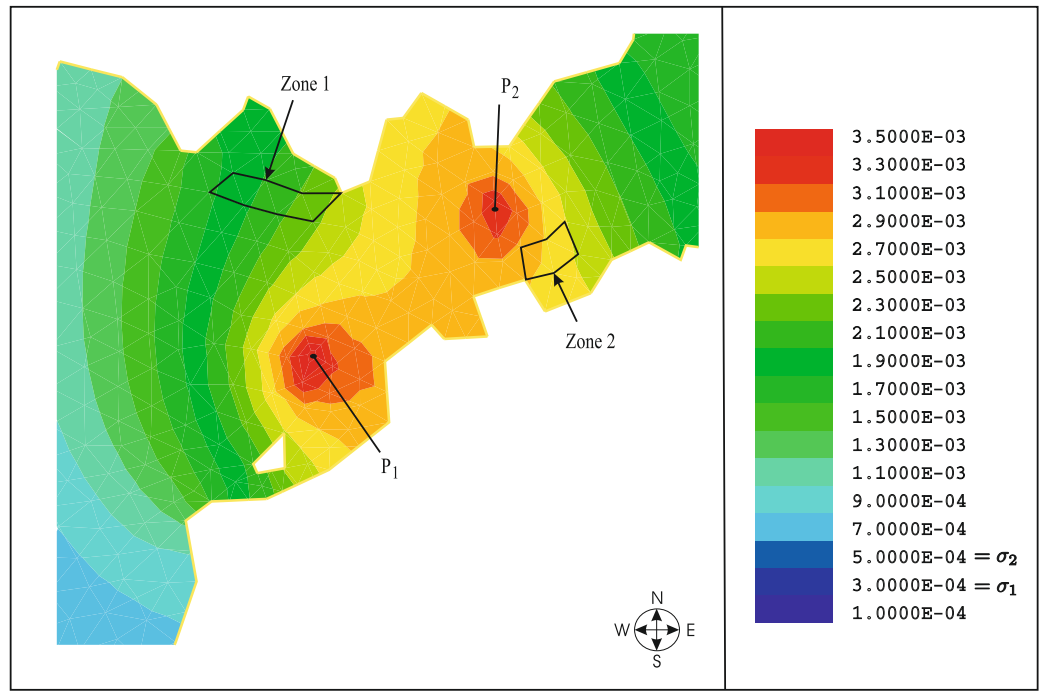

Figure 5. CF concentration in the neighborhood of protected areas at final time for $\epsilon_{1}=\epsilon_{2}=1$. 


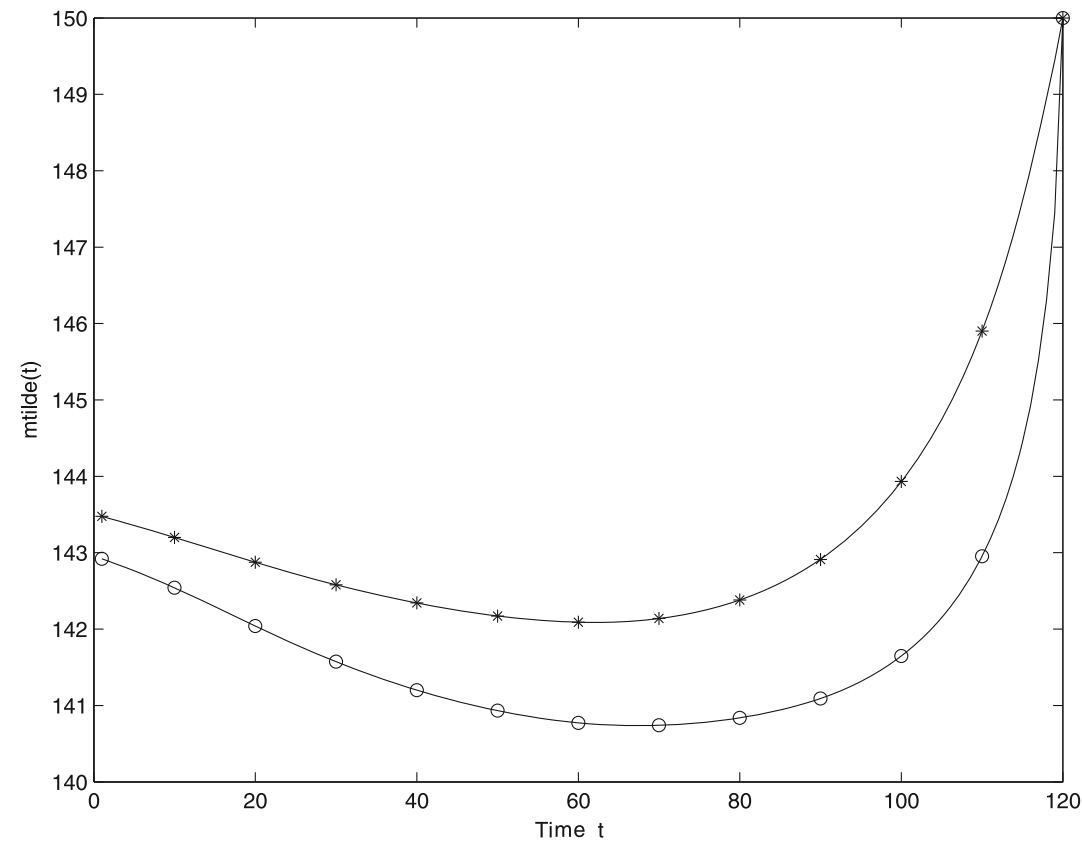

Figure 6. Optimal discharges $m_{1}^{*}(t)(* *)$ and $m_{2}^{*}(t)(o o)$ for $\epsilon_{1}=\epsilon_{2}=10^{-3}$.

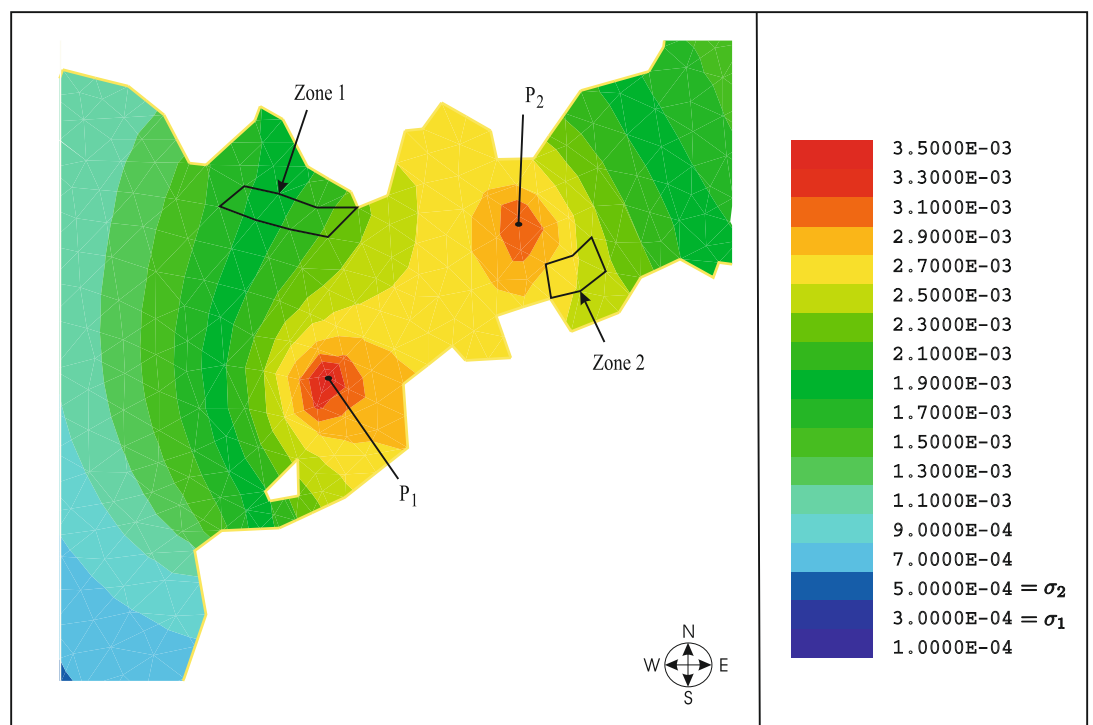

Figure 7. CF concentration in the neighborhood of protected areas at final time for $\epsilon_{1}=\epsilon_{2}=10^{-3}$. 


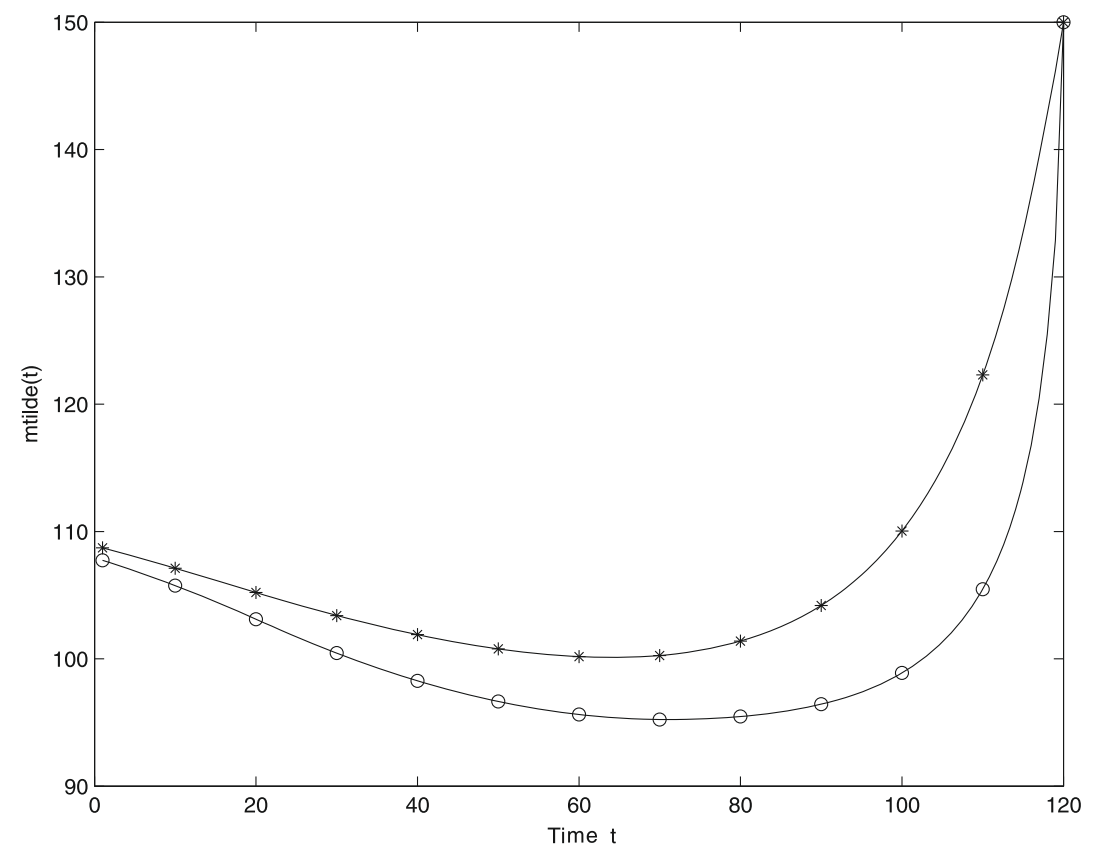

Figure 8. Optimal discharges $m_{1}^{*}(t)(* *)$ and $m_{2}^{*}(t)(o o)$ for $\epsilon_{1}=\epsilon_{2}=10^{-5}$.

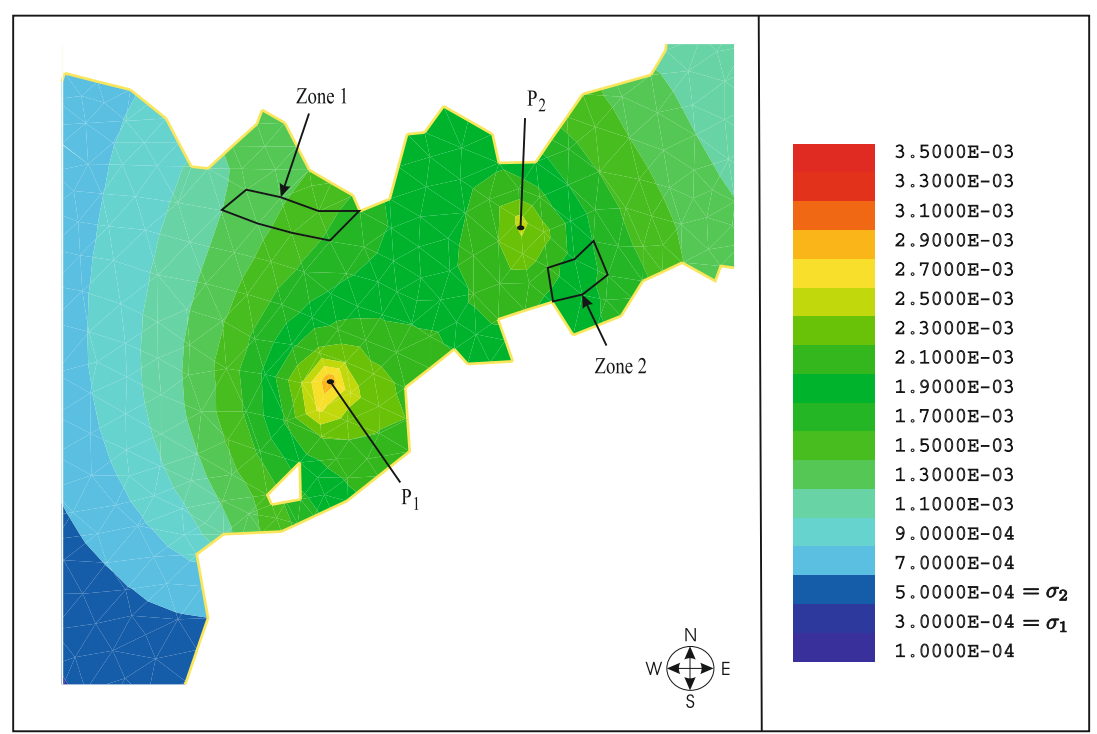

Figure 9. CF concentration in the neighborhood of protected areas at final time for $\epsilon_{1}=\epsilon_{2}=10^{-5}$. 


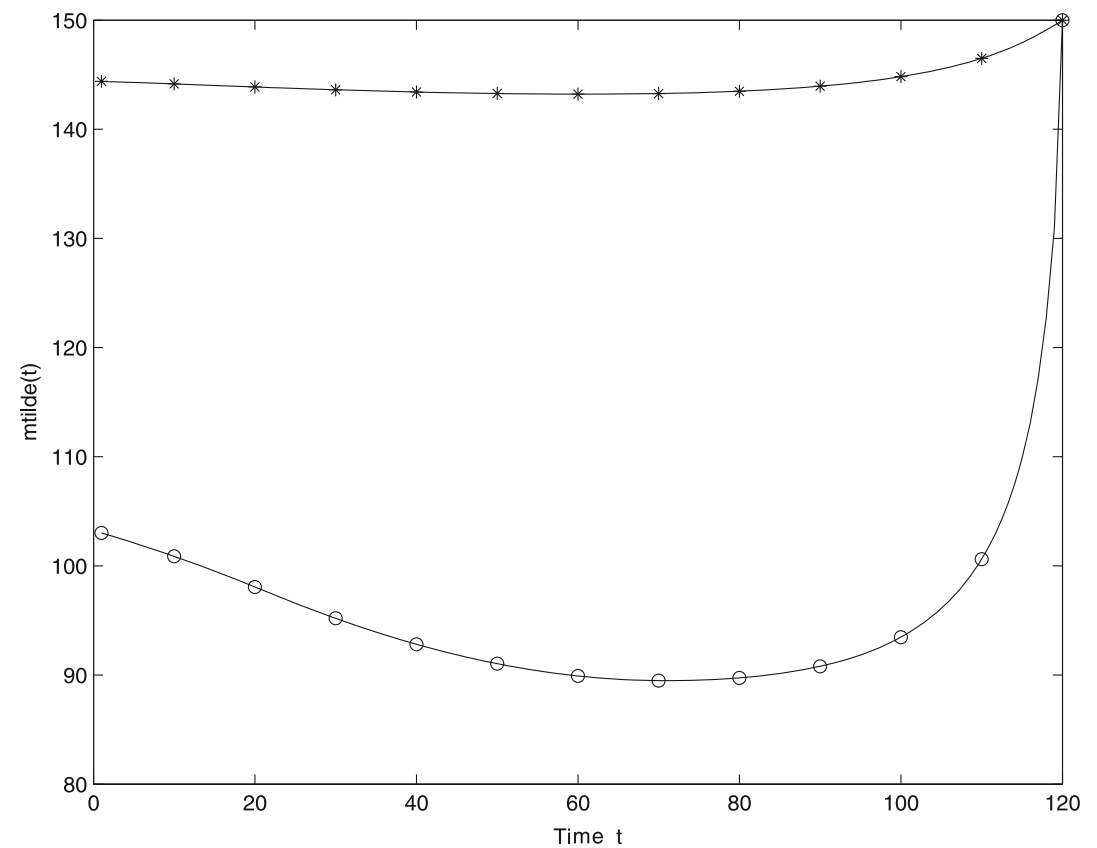

Figure 10. Optimal discharges $m_{1}^{*}(t)(* *)$ and $m_{2}^{*}(t)($ oo $)$ for $\epsilon_{1}=10^{-3}, \epsilon_{2}=10^{-5}$.

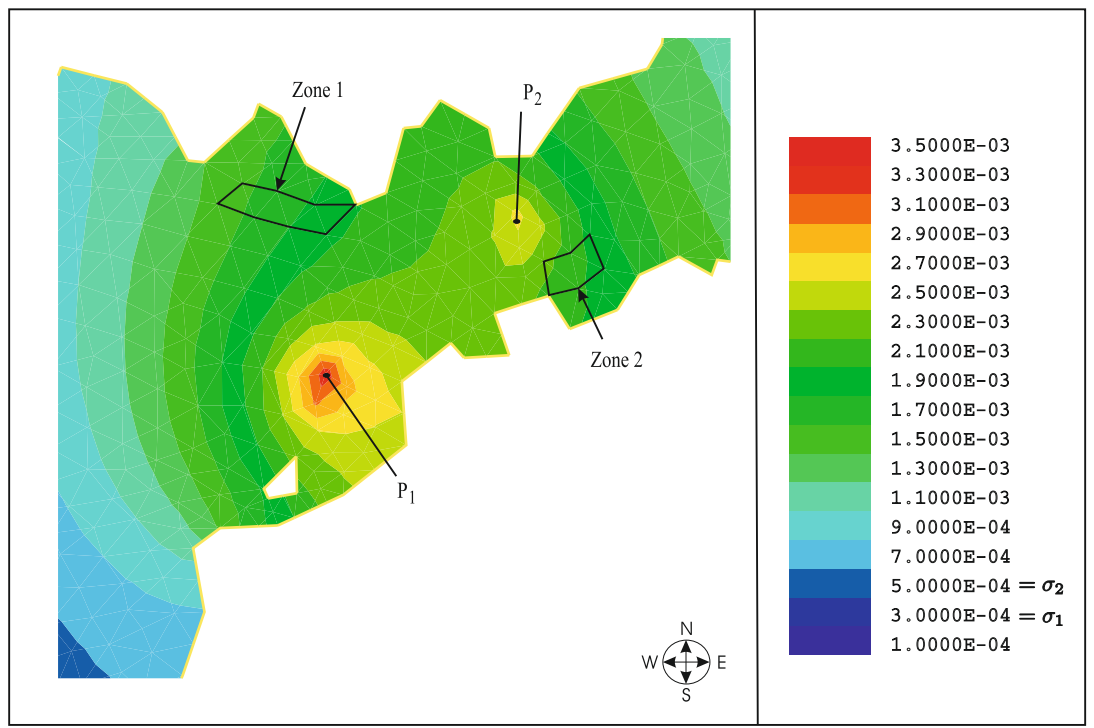

Figure 11. CF concentration in the neighborhood of protected areas at final time for $\epsilon_{1}=$ $10^{-3}, \epsilon_{2}=10^{-5}$. 


\section{REFERENCES}

[1] L.J. Álvarez-Vázquez, A. Martínez, C. Rodríguez and M.E. Vázquez-Méndez, Numerical convergence for a sewage disposal problem. Appl. Math. Model. 25 (2001) 1015-1024.

[2] L.J. Álvarez-Vázquez, A. Martínez, C. Rodríguez and M.E. Vázquez-Méndez, Numerical optimization for the location of wastewater outfalls. Comput. Optim. Appl. 22 (2002) 399-417.

[3] L.J. Álvarez-Vázquez, A. Martínez, C. Rodríguez and M.E. Vázquez-Méndez, Mathematical model for optimal control in wastewater discharges: the global performance. C. R. Biologies 328 (2005) 327-336.

[4] L.J. Álvarez-Vázquez, A. Martínez, R. Muñoz-Sola, C. Rodríguez and M.E. Vázquez-Méndez, The water conveyance problem: Optimal purification of polluted waters. Math. Models Meth. Appl. Sci. 15 (2005) 1393-1416.

[5] A. Bermúdez, Numerical modelling of water pollution problems, in Environment, Economics and their Mathematical Models, J.I. Diaz and J.L. Lions Eds., Masson, Paris (1994).

[6] A. Bermúdez, C. Rodríguez and M.A. Vilar, Solving shallow water equations by a mixed implicit finite element method. IMA J. Num. Anal. 11 (1991) 79-97.

[7] E. Casas, Pontryagin's principle for state constrained boundary control problems of semilinear parabolic equations. SIAM J. Control Optim. 35 (1997) 1297-1327.

[8] R. Gibbons, A Primer in Game Theory. Pearson Higher Education (1992).

[9] O.A. Ladyzenskaja, V.A. Solonnikov and N.N. Ural'ceva, Linear and quasilinear equations of parabolic type, in Translations of Mathematical Monographs 23, Amer. Math. Soc., Providence (1968).

[10] J.L. Lions, Contrôle optimal des systèmes gouvernés par des équations aux derivées partielles. Dunod, Paris (1968).

[11] J.L. Lions and E. Magenes, Problèmes aux limites non homogenes et applications. Dunod, Paris (1968).

[12] A. Martínez, C. Rodríguez and M.E. Vázquez-Méndez, Theoretical and numerical analysis of an optimal control problem related to wastewater treatment. SIAM J. Control Optim. 38 (2000) 1534-1553.

[13] D. Parra-Guevara and YN. Skiba, Elements of the mathematical modeling in the control of pollutants emissions. Ecol. Model. 167 (2003) 263-275.

[14] O. Pironneau, Finite Element Methods for Fluids. J. Wiley \& Sons, Chichester (1989).

[15] A.M. Ramos, R. Glowinski and J. Periaux, Nash equilibria for the multiobjetive control of linear partial differential equations. J. Optim. Theory Appl. 112 (2002) 457-498.

[16] E. Zeidler, Nonlinear Functional Analysis and its Applications. Springer-Verlag (1993). 